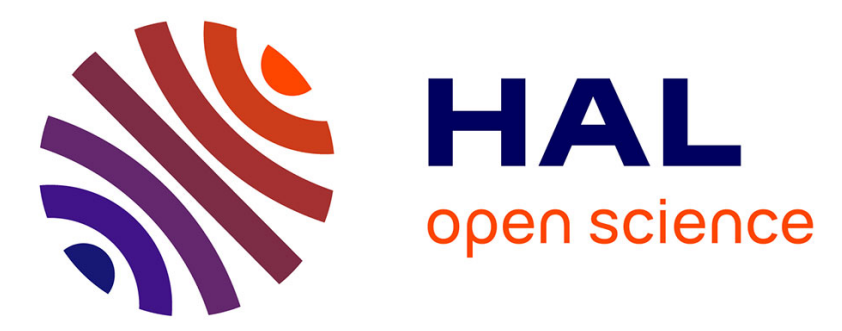

\title{
Mixture of consistent stochastic utilities, and a priori randomness
}

Mrad Mohamed

\section{To cite this version:}

Mrad Mohamed. Mixture of consistent stochastic utilities, and a priori randomness. International Journal of Theoretical and Applied Finance, 2020. hal-01728554v2

\section{HAL Id: hal-01728554 \\ https://hal.science/hal-01728554v2}

Submitted on 26 Nov 2020

HAL is a multi-disciplinary open access archive for the deposit and dissemination of scientific research documents, whether they are published or not. The documents may come from teaching and research institutions in France or abroad, or from public or private research centers.
L'archive ouverte pluridisciplinaire HAL, est destinée au dépôt et à la diffusion de documents scientifiques de niveau recherche, publiés ou non, émanant des établissements d'enseignement et de recherche français ou étrangers, des laboratoires publics ou privés. 


\title{
Mixture of consistent stochastic utilities, and a priori randomness *†
}

\author{
Mrad Mohamed $\ddagger$
}

November 25, 2020

\begin{abstract}
The purpose of this paper is to develop an explicit construction of consistent utilities, using the stochastic flows approach developed in El Karoui \& Mrad (2013, 2020). Starting from a family of utility functions indexed by some parameter $\alpha$ (for example the risk aversion coefficient or any other parameter), the idea is to randomize $\alpha$ and to construct non-standard stochastic utility processes. Two approaches are developed. The first one consists of building directly from the class $\left\{U^{\alpha}, \alpha \in \mathbb{R}\right\}$ a global utility $U$ as a supconvolution. The second approach which is very different, consists to define from the class $\left(X^{\alpha}, Y^{\alpha}\right)_{\alpha \in \mathbb{R}}$ of monotonic optimal processes, associated with the class $\left\{U^{\alpha}, \alpha \in \mathbb{R}\right\}$, a global pair $\left(X^{*}, Y^{*}\right)$ as a mixture. The non standard stochastic utility is then obtained by composing stochastic flows and is interpreted as the aggregate utility of all considered agents.
\end{abstract}

\section{Introduction}

The aim of this work is to build a large class of consistent utilities using ideas of stochastic change of variables introduced in El Karoui \& Mrad (2013, 2020) in a Itô's framework where both securities and dynamic utilities are modeled as continuous Itô's semimartingales. The concept of consistent stochastic utilities, also called "forward dynamic utilities", has been introduced by Musiela \& Zariphopoulou (2003, 2007, 2010, 2011) ; since then this notion appears

\footnotetext{
*This work was supported the Labex MME-DII .

${ }^{\dagger}$ Keywords: forward utility, performance criteria, horizon-unbiased utility, consistent utility, progressive utility, portfolio optimization, optimal portfolio, duality, minimal martingale measure, Stochastic flows, Stochastic partial differential equation.

‡LAGA, UMR CNRS 7539, Université Paris 13.
} 
in the literature in varied forms, in the work of Choulli et al. (2007), Henderson \& Hobson (2007), Berrier et al. (2009), Zitkovic (2008) and in El Karoui \& Mrad (2013). Intuitively, a stochastic utility should represent the individual preferences of an agent that are possibly changing over time. The agent's preferences are affected over time by the information available on the market represented by the filtration $\left(\mathcal{F}_{t}, t \geq 0\right)$ defined on the probability space $(\Omega, \mathbb{P}, \mathcal{F})$. The agent starts with today's specification of his utility, $U(0, x)=u(x)$, and then builds the process $U(t, x)$ for $t>0$ taking into account the information flow given by $\left(\mathcal{F}_{t}, t \geq 0\right)$. Consequently, the utility, denoted by $U(t, x)$ is a progressive process depending on time and wealth, $t$ and $x$, which is as a function of $x$ strictly increasing and concave. In contrast to the standard literature, there is no pre-specified trading horizon at which the utility is assigned. Consequently the initial function $u(x)$ is given in place of $U(T, x)$ where $T$ is the time horizon in the classical problem.

The results of this work can be applied to describe the behavior of a representative agent in an economy. Indeed, most of general equilibrium macroeconomic models are simplified by assuming that consumers and/or firms could be described as a representative agent. That is agents may differ and act differently, but at equilibrium the sum of their choices is mathematically equivalent to the decision of one individual. In the meantime, the existence of an equilibrium is not always satisfied and equilibrium are often stated and studied in a complete market setting. One key point for the existence of equilibrium is that agents agree on the same state price density process. This so called Pareto optimality is considered, to our knowledge for the first time with dynamic utilities, in Section 3. However, if no equilibrium exists, we would also like to propose a way of aggregating the preferences of heterogeneous investors in a given economy. This is developed in Sections 4 and 5.

In Section 2, we introduce the general framework of this work: the notion of progressive utilities, the consistent utility's definition and we recall some results, from El Karoui \& Mrad (2013, 2020), that will be used extensively in this paper. In Section 3, we give ourselves a family of consistent utility processes indexed by a parameter $\alpha,\left\{U^{\alpha}, \alpha \in \mathbb{R}\right\}$ and a finite positive Borel measure $m(d \alpha)$. Denoting by $\widetilde{U}^{\alpha}$ the dual convex conjugate of $U^{\alpha}$, we define the convex process $\widetilde{U}^{s}(t, y)=\int \widetilde{U}^{\alpha}(t, y) m(d \alpha)$ and show, assuming that all $\widetilde{U}^{\alpha}$ generate the same optimal state price density process denoted by $Y^{*}$ (Pareto-Optimality principle), that $\widetilde{U}^{s}$ is the dual convex conjugate of a consistent utility $U^{s}$. We show also, in Theorem 3.1, that $U^{s}(t, x)$ is the sup-convolution of the concave functions $U^{\alpha}(t, x)$ (see Barrieu \& El Karoui (2005) for similar result, with only two investors, in the standard framework of exponential utilities). Moreover, the optimal wealth process associated with $U^{s}$ is a mixture of optimal wealths $X^{*, \alpha}$ (associated with consistent utilities $\left.U^{\alpha}\right)$ starting from a given initial allocation $\left\{x^{\alpha}(x), \alpha\right\}$ of the global 
initial wealth $x$,

$$
X^{*}(t, x)=\int X^{*, \alpha}\left(t, x^{\alpha}(x)\right) m(d \alpha), \quad \text { with } \int x^{\alpha}(x) m(d \alpha)=x,
$$

where $u$ is the inverse Fenchel's transform of $\widetilde{U}^{s}(0, y)$. This observation is the starting point for the rest of the paper. Instead of aggregating utilities (not flexible enough and restrictive) we propose to aggregate portfolios and state prices and then generate new utilities using a general result obtained in El Karoui \& Mrad (2013, 2020). This idea will be developed in two stages in the rest of the paper. In Section 4.2, we give ourselves a finite positive Borel measure $m(d \alpha)$ and a family of deterministic utility functions $\left\{u^{\alpha}, \alpha \in \mathbb{R}\right\}$. In a first step, we generate, from $\left\{u^{\alpha}, \alpha \in \mathbb{R}\right\}$ (using techniques combining a change of numeraire and a change of probability) a new family of consistent utilities processes $\left\{U^{\alpha}, \alpha \in \mathbb{R}\right\}$ whose optimal processes (strictly increasing with respect to their initial conditions) are denoted by $X^{*, \alpha}$ and $Y^{*, \alpha}, \alpha \in \mathbb{R}$. As the processes $Y^{*, \alpha}, \alpha \in \mathbb{R}$ are not necessarily the same, we will rather consider another state density price $Y^{*}(y):=y Y^{*}$ such that $Y^{*} X^{*, \alpha}$ is a martingale for any $\alpha$. Thereafter, for a given family of strictly increasing positive functions $x^{\alpha}, \alpha \in \mathbb{R}$, satisfying $\int x^{\alpha}(x) m(d \alpha)=x$ for all $x$, the next step is then to build a new (monotonic) portfolio $X^{*}$, as a mixture of $X^{*, \alpha}, \alpha \in \mathbb{R}$, i.e.,

$$
X^{*}(t, x)=\int X^{*, \alpha}\left(t, x^{\alpha}(x)\right) m(d \alpha), X_{0}^{*}(x)=\int x^{\alpha}(x) m(d \alpha)=x .
$$

From this, after verifying that $X^{*} Y^{*}$ is a martingale and denoting by $\mathcal{X}$ the inverse of $X^{*}$ with respect to its initial condition, we generate a new consistent utility $U$ from any initial condition $u$ as follows

$$
U(t, x):=Y_{t}^{*} \int_{0}^{x} u_{x}(\mathcal{X}(t, z)) d z .
$$

As it is defined this consistent utility is not a mixture of utility processes $U^{\alpha}, \alpha \in \mathbb{R}$. Its initial condition $u$ could be any deterministic utility function, not necessarily equal to the supconvolution of initial functions $\left(u^{\alpha}\right)_{\alpha \in \mathbb{R}}$ as it is the case of Section 3. Moreover, $\left\{x^{\alpha}(),. \alpha \in \mathbb{R}\right\}$ in (1.2) are not necessarily Pareto-optimal. These last two points give us additional degrees of freedom and then one can generate a larger class of consistent utilities and one can aggregate more heterogeneous agents.

In Section 4.3, we show that the time-decreasing (zero volatility) consistent utilities fully characterized by Berrier et al. (2009) and Musiela \& Zariphopoulou (2010) are a very particular case of both constructions proposed in Sections 3 and 4.2. We also give a new proof of the main results of these papers, based on Mellin's transform and that exploits the linearity of the dual equation satisfied by the convex dual of time-decreasing dynamic utility. It is this linearity which is the key point of the aggregation. 
Finally, in Section 5, to provide a utility class even richer, the class $\left\{U^{\alpha}, \alpha \in \mathbb{R}\right\}$ is very general and similarly as for $X^{*}$ a dual process $Y^{*}$ is generated as a mixture of optimal processes $Y^{*, \alpha}$ for a given family of strictly increasing functions $y^{\alpha}$,

$$
Y^{*}(t, y)=\int Y^{*, \alpha}\left(t, y^{\alpha}(y)\right) m(d \alpha), Y_{0}^{*}(y)=\int y^{\alpha}(y) m(d \alpha)=y .
$$

The utility process proposed is then defined by

$$
U(t, x):=\int_{0}^{x} Y_{t}^{*}\left(u_{x}(\mathcal{X}(t, z))\right) d z
$$

for any utility function $u$ satisfying some integrability conditions.

\section{Preliminaries}

Utility function: Throughout this paper, we make the standard assumptions for a utility function $U$. A utility $U: \mathbb{R} \rightarrow \mathbb{R} \cup\{-\infty\}$ is increasing, continuous on $\{U>-\infty\}$, differentiable and strictly concave on the interior of $\{U>-\infty\}$ and such that $U_{x}$ goes to zero when the wealth $x$ goes to infinity, i.e.,

$$
U_{x}(\infty):=\lim _{x \rightarrow \infty} U_{x}(x)=0 .
$$

As regards the behavior of the (marginal) utility at the other end of the wealth scale we shall distinguish throughout the paper two cases.

Case 1 (negative wealth not allowed): in this setting we assume that $U$ satisfies the conditions $U(x)=-\infty$, for $x<0$, while $U(x)>-\infty$, for $x>0$, and that

$$
U_{x}(0):=\lim _{x \searrow 0} U_{x}(x)=\infty .
$$

Case 2 (negative wealth allowed): in this case we assume that $U(x)>-\infty$, for all $x \in \mathbb{R}$, and that

$$
U_{x}(-\infty):=\lim _{x \searrow-\infty} U_{x}(x)=\infty .
$$

In the first part of this work, we restrict ourselves only to the first case before considering the second one in Section 5.

Proper concave and convex functions: In convex analysis and optimization, a proper convex function is a convex function $f$ taking values in the extended real number line such that $f(x)<+\infty$ for at least one $x$ and $f(x)>-\infty$ for every $x$. That is, a convex function is proper if its effective domain is nonempty and if it never attains $-\infty$. A proper concave function is any function $g$ such that $f=-g$ is a proper convex function. 
Some properties of proper functions: Denote by $\tilde{f}$ the Fenchel's transform of $f$ defined by $\tilde{f}(y)=\max _{x \in \mathbb{Q}}(f(x)-x y)$.

(i) The convex conjugate $\tilde{f}$ of a lower semi-continuous (lsc) proper concave function $f$ is lsc proper convex function.

(ii) The infimal convolution (or epi-sum) of two functions $f$ and $g$ is defined as

$$
(f \square g)(x)=\inf _{y \in \mathbb{R}}\{f(x-y)+g(y)\} .
$$

Let $f_{1}, \ldots, f_{m}$ be proper, convex and lsc functions on $\mathbb{R}$. Then the infimal convolution is well defined convex and lsc (but not necessarily proper), and satisfies

$$
F:=\left(f_{1} \square \ldots \square f_{m}\right) \text { is such that } \tilde{F}=\tilde{f}_{1}+\ldots+\tilde{f}_{m} .
$$

Progressive utility and its Fenchel's conjugate We start with the definition of a progressive utility as progressive random field with concavity property.

Definition 2.1 (Progressive Utility). A progressive utility is a continuous progressive random field on $\mathbb{R}, \mathbf{U}=\{U(t, x) ; t \geq 0, x \in \mathbb{R}\}$ such that,

(i) UTILITY PROPERTY: U is strictly concave, strictly increasing, and non negative.

(ii) Regularity PROPERTy: $\mathbf{U}$ is a $\mathcal{C}^{2}$-random field, with continuous first and second derivatives random fields $\mathbf{U}_{\mathbf{x}}$ and $\mathbf{U}_{\mathbf{x x}}$.

(iii) InADA CONDITIOns: $\mathbf{U}$ goes to 0 when $x$ goes to 0 and the derivative $\mathbf{U}_{\mathbf{x}}$ goes to $\infty$ when $x$ goes to 0 , and to 0 when $x$ goes to $\infty$.

Note, if negative wealth are allowed, the Inada conditions become: $\mathbf{U}$ goes to 0 when $x$ goes to $-\infty$ and the derivative $\mathbf{U}_{\mathbf{x}}$ goes to $\infty$ when $x$ goes to $-\infty$.

Given its importance in convex analysis, we introduce together with any progressive utility $\mathbf{U}$, its convex conjugate $\widetilde{\mathbf{U}}$ (also called conjugate progressive utility (CPU)), that is the FenchelLegendre transform of the convex random field $-\mathbf{U}(.,-)$.

Definition 2.2 (Progressive conjugate utility). The convex conjugate of the progressive utility $\mathbf{U}$ is the progressive random field $\widetilde{\mathbf{U}}$ defined on $\mathbb{R}_{+}^{*}$ by $\widetilde{\mathbf{U}}=\{\widetilde{U}(t, y) ; t \geq 0, y>0\}$, where $\widetilde{U}(t, y) \stackrel{\text { def }}{=} \max _{x>0}(U(t, x)-x y)$.

(i) $\widetilde{\mathbf{U}}$ is twice continuously differentiable, strictly convex, strictly decreasing, with $\widetilde{U}\left(., 0^{+}\right)=$ $U(.,+\infty), \widetilde{U}(.,+\infty)=U\left(., 0^{+}\right)$, a.s.

(ii) The marginal utility $\mathbf{U}_{x}$ is the inverse of the opposite of the marginal conjugate utility $\widetilde{\mathbf{U}}_{y}$, that is $U_{x}(t, .)^{-1}(y)=-\widetilde{U}_{y}(t, y)$, with Inada conditions $\widetilde{U}_{y}\left(., 0^{+}\right)=-\infty, \widetilde{U}_{y}(.,+\infty)=0$.

(iii) The bi-dual relation holds true $U(t, x)=\inf _{y>0, y \in \mathbb{Q}^{+}}(\widetilde{U}(t, y)+x y)$. Moreover $\widetilde{U}(t, y)=U(t,-\widetilde{U}(t, y))+\widetilde{U}_{y}(t, y) y$, and $U(t, x)=\widetilde{U}\left(t, U_{x}(t, x)\right)+x U_{x}(t, x)$. 
The notion of progressive utility is very general and should be specified so as to represent more realistically the dynamic evolution of the individual preferences of an investor in a given financial market. The market input is described by a vector space of portfolios incorporating feasibility and trading constraints and high liquidity. We give ourselves, a sub-set $\mathscr{X}$ of portfolios (playing the role of learning class) to which we will calibrate the dynamic utility of an investor. We associate to this class the set $\mathscr{Y}$ of adjoint processes also called state density processes,

$$
\mathscr{Y}:=\{Y \geq 0: Y X \text { is a local martingale for any } X \in \mathscr{X}\} .
$$

Following Musiela \& Zariphopoulou (2003, 2004, 2007), a $\mathscr{X}$-consistent dynamic utility is defined as follows.

Definition 2.3 ( $\mathscr{X}$-consistent Utility). A $\mathscr{X}$-consistent stochastic utility process $U(t, x)$ is a positive random field with the following properties:

CONCAVITY ASSUMPTION : for $t \geq 0, x \mapsto U(t, x)$ is an increasing concave function, (in short utility function).

Consistency : For any test wealth process $X \in \mathscr{X}, \mathbb{E}\left(U\left(t, X_{t}\right)\right)<+\infty$ and

$$
\mathbb{E}\left(U\left(t, X_{t}\right) \mid \mathcal{F}_{s}\right) \leq U\left(s, X_{s}\right), \forall s \leq t \text {.a.s. }
$$

ExistenCE OF OPTIMAL WEALTh: For any initial wealth, there exists an optimal wealth process $X^{*} \in \mathscr{X}$, such that $X_{0}^{*}=x$, and $U\left(s, X_{s}^{*}\right)=\mathbb{E}\left(U\left(t, X_{t}^{*}\right) \mid \mathcal{F}_{s}\right) \forall s \leq t$.

The consistency property is the analog of the dynamic programming principle in the classical theory of backward expected utility maximization.

It is shown, in El Karoui \& Mrad $(2013,2020)$, that the convex conjugate $\widetilde{U}$ of a consistent utility $U$ is consistent with the family $\mathscr{Y}$ of state price density processes in the following sense: for any $Y \in \mathscr{Y}$ the process $\widetilde{U}\left(t, Y_{t}\right)$ is a submartingale and martingale for an optimal choice $Y_{t}^{*}(y)=U_{x}\left(t, X_{t}^{*}\left(-\widetilde{U}_{y}(0, y)\right)\right) . \widetilde{U}(t, y)$ is said to be a $\mathscr{Y}$-consistent stochastic dual utility.

Notation: When the utility random field is defined on $[0,+\infty]$, the set $\mathscr{X}$ consists only of positive processes, it will be denoted $\mathscr{X}^{+}$.

\subsection{The power consistent stochastic utilities}

Power utilities are widely considered in the literature by economists because they allow to carry out calculations and thus obtain closed formulas for the optimal processes. Moreover, in an economic equilibrium, as the one described by He \& Leland (1993), a utility function can only be a mixture of power utilities, otherwise the equilibrium can not exist, see El Karoui \& Mrad (2020). 
In this section, we look for $\mathscr{X}^{+}$-consistent power utilities $U^{(\alpha)}(t, x)=Z_{t}^{(\alpha)} \frac{x^{1-\alpha}}{1-\alpha}$ where $\alpha$ is the risk aversion coefficient and $Z^{(\alpha)}$ is a semimartingale which is calibrated to satisfy the consistency property. As in the deterministic framework, the conjugate function $\widetilde{U}^{(\alpha)}(t, y)$ is given by $\widetilde{U}^{(\alpha)}(t, y)=-\tilde{Z}_{t}^{(\alpha)} \frac{y^{1-\frac{1}{\alpha}}}{1-\frac{1}{\alpha}}$ with $\tilde{Z}_{t}^{(\alpha)}=\left(Z_{t}^{(\alpha)}\right)^{\frac{1}{\alpha}}$.

Thanks to the consistency property, there exists an optimal portfolio $X_{t}^{(a), *}(x)$ such that

$$
U^{(\alpha)}\left(t, X_{t}^{(\alpha), *}(x)\right)=\frac{1}{1-\alpha} Z_{t}^{(\alpha)}\left(X_{t}^{(\alpha), *}(x)\right)^{1-\alpha} \text { is a martingale, }
$$

and s.t. $U_{x}\left(t, X_{t}^{(\alpha), *}(x)\right)=Y_{t}^{(\alpha), *}\left(x^{-\alpha}\right)$ is a state price density process with initial condition $x^{-\alpha}$. In particular, using the intuitive factorization $Z_{t}^{(\alpha)}=Z_{t}^{(\alpha, \sigma)} \cdot Z_{t}^{(\alpha, \perp)}$ where $Z_{t}^{(\alpha, \perp)}$ is a martingale s.t. $\left\langle Z^{(\alpha, \sigma)}, Z^{(\alpha, \perp)}>=<X^{(\alpha), *}, Z^{(\alpha, \perp)}>=0\right.$, we see that $Z_{t}^{(\alpha, \sigma)}\left(X_{t}^{(\alpha), *}(x)\right)^{-\alpha}=x^{-\alpha} Y_{t}^{0}$, where $Y_{t}^{0}$ is the minimal state price density. The optimal wealth $X_{t}^{(\alpha), *}(x)$ is linear with respect to its initial condition: $X_{t}^{(\alpha), *}(x)=x X_{t}^{(\alpha), *}(1)$ where $X_{t}^{(\alpha), *}(1)$ also denoted $\bar{X}_{t}^{(\alpha), *}$ is the optimal portfolio starting from $x=1$. Consequently,

$$
(\mathcal{I})\left\{\begin{aligned}
Z_{t}^{(\alpha)} & =Z_{t}^{(\alpha, \perp)} Y_{t}^{0}\left(\bar{X}_{t}^{(\alpha), *}\right)^{\alpha}, & & \tilde{Z}_{t}^{(\alpha)}=\bar{X}_{t}^{(\alpha), *}\left(\bar{Y}_{t}^{(\alpha), *}\right)^{\frac{1}{\alpha}} \\
X_{t}^{(\alpha), *}(x) & =x \bar{X}_{t}^{(\alpha), *}, & & Y_{t}^{(\alpha), *}(y)=y Z_{t}^{(\alpha, \perp)} Y_{t}^{0} \\
U^{(\alpha)}(t, x) & =\frac{1}{1-\alpha} \bar{Y}_{t}^{(\alpha), *} \bar{X}_{t}^{(\alpha), *}\left(\frac{x}{\bar{X}_{t}^{(\alpha), *}}\right)^{1-\alpha}, & & \widetilde{U}^{(\alpha)}(t, y)=-\frac{\bar{Y}_{t}^{(\alpha), *} \bar{X}_{t}^{(\alpha), *}}{1-\frac{1}{\alpha}}\left(\frac{y}{\bar{Y}_{t}^{(\alpha), *}}\right)^{1-\frac{1}{\alpha}}
\end{aligned}\right.
$$

where we have used the fact that $Y^{(a), *}(y)=y \bar{Y}^{(a), *}$.

Remark: Identities $X_{t}^{(\alpha), *}(x)=x \bar{X}_{t}^{(\alpha), *}$ and $Y_{t}^{(\alpha), *}(y)=y \bar{Y}_{t}^{(\alpha), *}$ show that these optimal processes are increasing in $x$ and $y$ with inverse flows $\mathcal{X}_{t}^{(\alpha)}(x)=x / \bar{X}_{t}^{(\alpha), *}$ and $\mathcal{Y}_{t}^{(\alpha)}(y)=y / \bar{Y}_{t}^{(\alpha), *}$. From this point, it is straightforward to check that $U^{(\alpha)}(t, x)$ and $\widetilde{U}^{(\alpha)}(t, y)$, taking $u(x)=\frac{x^{1-\alpha}}{1-\alpha}$, have the following representations,

$$
U^{(\alpha)}(t, x)=\int_{0}^{x} Y_{t}^{(\alpha), *}\left(u_{x}\left(\mathcal{X}_{t}^{(\alpha)}(z)\right)\right) d z, \quad \widetilde{U}^{(\alpha)}(t, y)=\int_{y}^{+\infty} X_{t}^{(\alpha), *}\left(-\tilde{u}_{y}\left(\mathcal{Y}_{t}^{(\alpha)}(z)\right)\right) d z .
$$

Note, in particular, that $Y_{t}^{(\alpha), *}\left(u_{x}(x)\right)=U_{x}^{(\alpha)}\left(t, X_{t}^{(\alpha), *}(x)\right)$.

The next section comes back into details to this interesting representation of stochastic utility and its dual convex. We recall also, as established in El Karoui \& Mrad (2013, 2020), that this characterization is not specific to the power-type utilities.

\subsection{Main recent results characterizing consistent utilities}

In the theory of dynamic utilities, the first characterizations of these random fields were obtained by Berrier et al. (2009) and Musiela \& Zariphopoulou (2010, 2011) in the particular case where the volatility of the utility is zero and such utilities satisfy standard PDE. Later, a general characterization is given by El Karoui \& Mrad (2013) using stochastic characteristics method 
to solve the utility SPDE, for which classical result not apply. A direct approach, in abstract form, is developed in El Karoui \& Mrad (2020) in which minimal regularity assumptions on processes are done and general results are obtained by methods of analysis and compounding maps without stochastic calculus.

In El Karoui \& Mrad (2013, 2020), the dual problem has been considered with the same attention as the primal one. This highlight the role played by the processes $U_{x}\left(t, X_{t}^{*}\right)$ and especially this emphasizes the perfect symmetry between the primal problem whose optimum is $X^{*}$ and the dual one whose optimum is $Y_{t}^{*}\left(u_{x}(x)\right)=U_{x}\left(t, X_{t}^{*}(x)\right)$. In particular, this naturally leads to the next result (Theorem 2.1) characterizing these utilities from the optimality condition combined with the monotonicity of $X^{*}$ and $Y^{*}$ with respect to their initial conditions. Furthermore, it is sometimes easier to consider the dual convex $\widetilde{U}$ than the utility $U$, as it is the case of the decreasing stochastic utilities recalled in Section 4.3.

The following theorem summarizes a series of results established in El Karoui \& Mrad (2013, 2020). It gives the necessary and sufficient condition of consistency and existence of an optimum. It also gives sufficient conditions that we meet more often in the context of finance and economics.

Theorem 2.1. Let $\left(X^{*}, Y^{*}\right)$ be a pair of a wealth process $X^{*} \in \mathscr{X}^{+}$and a state density price process $Y^{*} \in \mathscr{Y}$ assumed to be continuous and increasing resp. in $x$ and $y$ from 0 to $+\infty$ s.t. $X_{t}^{*}(0)=Y_{t}^{*}(0)=0, \quad X_{t}^{*}(+\infty)=Y_{t}^{*}(+\infty)=+\infty$ a.s. for any $t$. Denote by $\mathcal{X}$ and $\mathcal{Y}$ the inverse flows of $X^{*}$ and $Y^{*}$. Let $u$ be a deterministic utility function and consider the random field $U$ defined by

$$
U(t, x)=\int_{0}^{x} Y_{t}^{*}\left(u_{x}(\mathcal{X}(t, z))\right) d z .
$$

Necessary and sufficient condition: $U$ is well defined and is a $\mathscr{X}^{+}$-consistent stochastic utility with optimal portfolio $X^{*}$ and optimal state density price $Y^{*}$ if and only if for any $\left(x, x^{\prime}\right), x<x^{\prime}$, there exists an optional process $\psi_{t}\left(x, x^{\prime}\right)$ taking values in the interval $\left(x, x^{\prime}\right)$, such that the process $\left(X_{t}^{*}\left(x^{\prime}\right)-X_{t}^{*}(x)\right) Y_{t}^{*}\left(u_{x}\left(\psi_{t}\left(x, x^{\prime}\right)\right)\right)$ is a martingale.

Moreover, the dual convex conjugate of $U$ is given by

$$
\widetilde{U}(t, y)=\int_{y}^{+\infty} X_{t}^{*}\left(-\tilde{u}_{y}(\mathcal{Y}(t, z))\right) d z
$$

Remark 2.1. The necessary and sufficient condition is satisfied if one of the following statements holds

(i) $\left\{Y_{t}^{*}\left(u_{x}(x)\right)\left(X_{t}^{*}\left(x^{\prime}\right)-X_{t}^{*}(x)\right)\right\}$ is a supermartingale $\forall x>0, x^{\prime}>0$.

(ii) $X^{*}(x)$ is differentiable and $\left\{X_{x}^{*}(t, x) Y_{t}\left(u_{x}(x)\right)\right\}$ is a martingale for any $x>0$. 
(iii) Strong orthogonality: $\left\{X_{t}^{*}(x) Y_{t}(y)\right\}$ is a martingale for any $x>0, y>0$.

The sufficient conditions (ii) and (iii) are the most useful.

Note that in this theorem no regularity in time is required. Indeed, these results are valid in a general framework covering processes with jumps and can even apply in a discrete frame to learn the utility of an agent. Moreover, the application of these results goes well beyond the scope of finance and economics because they are based solely on properties of monotonicity and process orthogonality.

\section{Sup-Convolution of $\mathscr{X}$-consistent Utilities}

The first aggregation method that I propose is the sup-convolution, based on the principle of Pareto optimality which is one of the most widely used principles in economics and more precisely in the theory of economic equilibrium, see for example the original paper Pareto (1913) and also Censor (1977), Majumdar et al. (1980) and Migdalas et al. (2008). Closer to our approach see Barrieu \& El Karoui (2005) for a case of two agents with exponential utility functions, but to our knowledge this question has never been addressed for dynamic utilities. Let $\mathscr{X}$ to be any set of wealth processes and we are interested in mixtures of $\mathscr{X}$-consistent utilities that are still $\mathscr{X}$-consistent. For instance, we want to evaluate the gain of diversification over the different $K$ business units of a financial firm, modeled by different stochastic utilities $U^{i}(t, x)$ assumed to be proper concave functions, for example with different risk aversion coefficients. Remark that these utilities are not necessarily of the same type (the case where the utilities are all of the same type, for example a power or exponential type, is a particular case).

The goal is to find the fair allocation of the wealth $x$ between the different units, in the following sense: find $\left(x_{1}^{*}, x_{2}^{*} \ldots, x_{K}^{*}\right)$ with $\sum_{1}^{K} x_{i}^{*}=x$ such that

$$
U^{s}(t, x)=\sup \left\{\sum_{i=1}^{K} U^{i}\left(t, x_{i}\right) \mid \forall i x_{i} \geq 0, \text { and } \sum_{i=1}^{K} x_{i}=x\right\}
$$

achieves its maximum on $\left(x_{1}^{*}, x_{2}^{*} \ldots, x_{K}^{*}\right)$. We then study the aggregate progressive utility $U^{s}{ }^{1}$. In particular, we are looking for sufficient conditions under which $U^{s}$ is an $\mathscr{X}$-consistent utility. In convex analysis, the utility $U^{s}(t, x)$ is known as the sup-convolution of the concave functions $U^{i}(t, x)$. Such utilities are easier to study from the dual point of view since $\widetilde{U}^{s}(y)=\sum_{1}^{K} \widetilde{U}^{i}(t, y)$. The same problem may be extended to a continuous set of units $U^{\theta}$ with a positive finite measure $m(d \theta)$.

$$
\widetilde{U}^{s}(t, y)=\int \widetilde{U}^{\theta}(t, y) m(d \theta) .
$$

\footnotetext{
${ }^{1}$ Assuming the functions $U^{\theta}(t,$.$) to be proper guarantees that U^{s}(t,$.$) is well defined by (3.1).$
} 
Mixture of convex dual utilities and sup-convolution Consider a family $\widetilde{U}^{\theta}(t, y)$ of convex $\mathscr{X}$-consistent dual utilities ( $U^{\theta}$ the associated primal functions) and define $\widetilde{U}^{s}(t, y)=$ $\int \widetilde{U}^{\theta}(t, y) m(d \theta)$. Denote by $\tilde{u}()=.\widetilde{U}^{s}(0,$.$) and by u$ its inverse Fenchel's transform.

Assumption 3.1. Assume that at any time $t$, the convex functions $\widetilde{U}^{\theta}(t,$.$) are proper contin-$ uously differentiable such that $y \mapsto \widetilde{U}^{s}(t, y)$ is continuously differentiable with first derivative $\widetilde{U}_{y}^{s}(t, y)=\int \widetilde{U}_{y}^{\theta}(t, y) m(d \theta)$.

As a consequence, for any state price density process $Y^{\nu}, \widetilde{U}^{s}\left(t, Y_{t}^{\nu}\right)=\int \widetilde{U}^{\theta}\left(t, Y_{t}^{\nu}\right) m(d \theta)$ is a submartingale, as a sum of positive submartingales and the martingale property can be achieved only for a process $Y_{t}^{*}$ such that $\widetilde{U}^{\theta}\left(t, Y_{t}^{*}\right)$ is a martingale, for any $\theta$. In other words, $Y^{*}$ must be a common optimal dual process.

By the first order optimality condition, $X_{t}^{*}(x)=-\widetilde{U}_{y}^{s}\left(t, Y_{t}^{*}\left(u_{x}(x)\right)\right)$, it follows

$$
X_{t}^{*}(x)=-\int \widetilde{U}_{y}^{\theta}\left(t, Y_{t}^{*}\left(u_{x}(x)\right)\right) m(d \theta) .
$$

Denoting by $X^{*, \theta}$ the optimal wealth generated by $U^{\theta}$, since $Y^{*}$ is the optimal state price density process for all the consistent utilities $U^{\theta}$, we have $X_{t}^{*, \theta}(x)=-\widetilde{U}_{y}^{\theta}\left(t, Y_{t}^{*}\left(u_{x}^{\theta}(x)\right)\right)$. From this, it is easy to check that

$$
-\widetilde{U}_{y}^{\theta}\left(t, Y_{t}^{*}\left(u_{x}(x)\right)\right)=X_{t}^{\theta}\left(\left(u_{x}^{\theta}\right)^{-1}\left(u_{x}(x)\right)\right),
$$

and thus $X^{*}$ is the aggregation of optimal wealths $X^{*, \theta}$,

$$
X^{*}(t, x)=\int X^{*, \theta}\left(t, x^{\theta}(x)\right) m(d \theta), \quad \text { for } x^{\theta}(x)=\left(u_{x}^{\theta}\right)^{-1}\left(u_{x}(x)\right) .
$$

One of the main result of this paper is the following.

Theorem 3.1. Let $\left\{\widetilde{U}^{\theta}(t, y), \theta\right\}$ be a family of proper convex dual utilities. If Pareto-optimality holds then necessarily they have the same optimal process $Y^{*}$. Moreover,

(i) The utility process $U^{s}$ with the dual transform $\widetilde{U}^{s}(t, y)=\int \widetilde{U}^{\theta}(t, y) m(d \theta)$ is given as the Sup-Convolution of $U^{\theta}$ :

$$
U^{s}(t, x)=\sup \left\{\int U^{\theta}\left(t, x^{\theta}(x)\right) m(d \theta) ; \quad \int x^{\theta}(x) m(d \theta)=x\right\} .
$$

(ii) The supremum is achieved at the family

$$
\left\{\hat{x}^{\theta}(t, x):=\left(U_{x}^{\theta}\right)^{-1}\left(t,-\left(\widetilde{U}_{y}^{s}\right)^{-1}(t, x)\right), \theta\right\} \text { satisfying } \int \hat{x}^{\theta}(t, x) m(d \theta)=x, \forall t .
$$

(iii) $U^{s}$ is a $\mathscr{X}$-consistent stochastic utility with optimal wealth

$$
X^{*}(t, x)=\int X_{t}^{*, \theta}\left(\hat{x}^{\theta}(0, x)\right) m(d \theta)
$$

Moreover, for any $\theta$, we have the identity $\hat{x}^{\theta}\left(t, X_{t}^{*}(x)\right)=X_{t}^{*, \theta}\left(\hat{x}^{\theta}(0, x)\right)$. 
This result extends the one established in El Karoui \& Mrad (2020), for a mixture of power utilities with constant relative risk aversion $\theta$.

Proof. (i) and (ii): As it is defined, the random field $U^{s}(t, x)$ is given by

$$
U^{s}(t, x):=\inf _{y>0}\left\{\widetilde{U}^{s}(t, y)+x y\right\}=\inf _{y>0}\left\{\int \widetilde{U}^{\theta}(t, y) m(d \theta)+x y\right\} .
$$

In particular for any family of functions $x^{\theta}(x): \int x^{\theta}(x) m(d \theta)=x, U^{s}(t, x)$ can be written as

$$
U^{s}(t, x)=\inf _{y>0}\left\{\int\left(\widetilde{U}^{\theta}(t, y)+y x^{\theta}(x)\right) m(d \theta)\right\} .
$$

Additionally, as $\widetilde{U}^{\theta}$ is the convex conjugate of $U^{\theta}$, we have

$$
\widetilde{U}^{\theta}(t, y)+y x^{\theta}(x) \geq U^{\theta}\left(t, x^{\theta}(x)\right) .
$$

Plugging this into (3.10), leads to

$$
U^{s}(t, x) \geq \int U^{\theta}\left(t, x^{\theta}(x)\right) m(d \theta) \text { with } \int x^{\theta}(x) m(d \theta)=x .
$$

Therefore

$$
U^{s}(t, x) \geq \sup _{x^{\theta}: \int x^{\theta}(x) m(d \theta)=x} \int U^{\theta}\left(t, x^{\theta}(x)\right) m(d \theta) .
$$

To conclude it remains to establish the equality. First from Assumption 3.1, using

$$
\left(U_{x}^{s}\right)^{-1}(t, y)=-\widetilde{U}_{y}^{s}(t, y) \text { and }\left(U_{x}^{\theta}\right)^{-1}(t, y)=-\widetilde{U}_{y}^{\theta}(t, y)
$$

it follows that $\int\left(U_{x}^{\theta}\right)^{-1}\left(U_{x}^{s}(x)\right) m(d \theta)=x$ so that the family $\left\{\hat{x}^{\theta}(t, x):=\left(U_{x}^{\theta}\right)^{-1}\left(t, U_{x}^{s}(t, x)\right), \theta\right\}$ satisfies the condition

$$
\int \hat{x}^{\theta}(t, x) m(d \theta)=x
$$

Second, using the dual identity

$$
U^{\theta}(t, x)=\widetilde{U}^{\theta}\left(t, U_{x}^{\theta}(t, x)\right)+x U_{x}^{\theta}(t, x),
$$

it follows that

$$
\begin{aligned}
U^{\theta}\left(t, \hat{x}^{\theta}(t, x)\right) & =\widetilde{U}^{\theta}\left(t, U_{x}^{\theta}\left(t,\left(U_{x}^{\theta}\right)^{-1}\left(t, U_{x}^{s}(t, x)\right)\right)\right) \\
& +\left(U_{x}^{\theta}\right)^{-1}\left(t, U_{x}^{s}(t, x)\right) U_{x}^{\theta}\left(t,\left(U_{x}^{\theta}\right)^{-1}\left(t, U_{x}^{s}(t, x)\right)\right) \\
& =\widetilde{U}^{\theta}\left(t, U_{x}^{s}(t, x)\right)+U_{x}^{s}(t, x)\left(U_{x}^{\theta}\right)^{-1}\left(t, U_{x}^{s}(t, x)\right) \\
& =\widetilde{U}^{\theta}\left(t, U_{x}^{s}(t, x)\right)+U_{x}^{s}(t, x) \hat{x}^{\theta}(t, x) .
\end{aligned}
$$


Integrating with respect to the parameter $\theta$, yields

$$
\begin{aligned}
\int U^{\theta}\left(t, \hat{x}^{\theta}(t, x)\right) m(d \theta) & =\int \widetilde{U}^{\theta}\left(t, U_{x}^{s}(t, x)\right) m(d \theta)+U_{x}^{s}(t, x) \int \hat{x}^{\theta}(t, x) m(d \theta) \\
& \stackrel{\text { (3.2) and }}{=}{ }^{(3.14)} \widetilde{U}^{s}\left(t, U_{x}^{s}(t, x)\right)+x U_{x}^{s}(t, x)=U^{s}(t, x) .
\end{aligned}
$$

Combining this with (3.12) yields $(i)$ and (ii).

Let now focus on assertion (iii): by assumption $\widetilde{U}$ is the convex conjugate of $U^{s}$, which is consistent with the family of state density processes $\mathcal{Y}$, and achieves its maximum on $Y^{*}$ also optimal for all utilities $\widetilde{U}^{\theta}$. This leads, by analogy between the dual and primal problem, to the consistency of $U^{s}$ as explained above. We have now to show the optimality of the process $X_{t}^{*}:=\int X^{*, \theta}\left(t, \hat{x}^{\theta}(x)\right) m(d \theta)$ i.e., the martingale property of $U^{s}\left(t, X_{t}^{*}\right)$.

At first, by the first order optimality condition, the optimal primal process $X_{t}^{*}(x)$ is necessarily equal to $-\widetilde{U}_{y}^{s}\left(t, Y_{t}^{*}\left(U^{s}(0, x)\right)\right)$. As $Y^{*}=Y^{*, \theta}, \forall \theta$, once again by the optimality first order condition it follows that the individual optimal wealth processes are given by

$$
X_{t}^{*, \theta}(x)=-\widetilde{U}_{y}^{\theta}\left(t, Y_{t}^{*}\left(U_{x}^{\theta}(0, x)\right)\right)
$$

Consequently,

$$
\begin{aligned}
X_{t}^{*}(x) & =-\widetilde{U}_{y}^{s}\left(t, Y_{t}^{*}\left(U^{s}(0, x)\right)\right):=-\int \widetilde{U}_{y}^{\theta}\left(t, Y_{t}^{*}\left(U^{s}(0, x)\right)\right) m(d \theta) \\
& =\int X_{t}^{*, \theta}\left(-\widetilde{U}_{y}^{\theta}\left(0, U_{x}^{s}(0, x)\right)\right) m(d \theta)=\int X_{t}^{*, \theta}\left(x^{\theta}(x)\right) m(d \theta)
\end{aligned}
$$

Now, the dual identity and the optimality of $Y^{*}$ lead to

$$
\begin{aligned}
U^{s}\left(t, X_{t}^{*}(x)\right) & \left.=\widetilde{U}^{s}\left(t,-\widetilde{U}_{y}^{s}\left(t, X_{t}^{*}(x)\right)\right)+\widetilde{U}_{y}^{s}\left(t, X_{t}^{*}(x)\right) X_{t}^{*}(x)\right) \\
& \left.=\widetilde{U}^{s}\left(t, Y_{t}^{*}\left(-\widetilde{U}_{y}^{s}(0, x)\right)\right)+Y_{t}^{*}\left(-\widetilde{U}_{y}^{s}(0, x)\right) X_{t}^{*}(x)\right)
\end{aligned}
$$

which implies, since it is the sum of two martingales, that $\left(U^{s}\left(t, X_{t}^{*}(x)\right)\right)_{t}$ is a martingale. To achieve the proof, let us show the identity $\hat{x}^{\theta}\left(t, X_{t}^{*}(x)\right)=X_{t}^{*, \theta}\left(\hat{x}^{\theta}(0, x)\right)$. By definition $\hat{x}^{\theta}(t, x):=\left(U_{x}^{\theta}\right)^{-1}\left(t, U_{x}^{s}(t, x)\right)$. Using the identities

$$
\left\{\begin{array}{c}
U_{x}^{s}\left(t, X_{t}^{*}(x)\right)=Y_{t}^{*}\left(U_{x}^{s}(0, x)\right)=U_{x}^{\theta}\left(t, X^{*, \theta}\left(-\widetilde{U}_{y}^{\theta}\left(0, U_{x}^{s}(0, x)\right)\right)\right) \\
\hat{x}^{\theta}(0, x):=-\widetilde{U}_{y}^{\theta}\left(0, U_{x}^{s}(0, x)\right)
\end{array}\right.
$$

it follows

$$
\begin{aligned}
\hat{x}^{\theta}\left(t, X_{t}^{*}(x)\right) & =-\widetilde{U}_{y}^{\theta}\left(t, U_{x}^{s}\left(t, X^{*}(t, x)\right)\right)=-\widetilde{U}_{y}^{\theta}\left(t, U_{x}^{\theta}\left(t, X^{*, \theta}\left(-\widetilde{U}_{y}^{\theta}\left(0, U_{x}^{s}(0, x)\right)\right)\right)\right) \\
& =X_{t}^{*, \theta}\left(-\widetilde{U}_{y}^{\theta}\left(0, U_{x}^{s}(0, x)\right)=X_{t}^{*, \theta}\left(\hat{x}^{\theta}(0, x)\right) .\right.
\end{aligned}
$$


Example Consider the case of power utilities studied in Section 2.1 where the parameter $\theta$ plays the role of the relative risk aversion $\alpha$ : $U^{\alpha}(t, x)=Z_{t}^{(\alpha)} \frac{x^{1-\alpha}}{1-\alpha}$. Taking $Z_{t}^{(\alpha), \perp} \equiv 1, \forall \alpha$, the state price density $Y^{*}=Y^{0}$ is optimal for all $U^{\theta}$. According to the system $(\mathcal{I})$, one easily gets, in this case, that the convex conjugate $\widetilde{U}^{s}$ of the consistent utility $U^{s}$ is given by

$$
\widetilde{U}^{s}(t, y)=\int-\frac{1}{1-\frac{1}{\alpha}} Y_{t}^{0} X_{t}^{(\alpha)}\left(y / Y_{t}^{0}\right)^{1-\frac{1}{\alpha}} m(d \alpha),
$$

with the deterministic initial dual utility

$$
\widetilde{U}^{s}(0, y)=\tilde{u}^{s}(y)=\int-\frac{1}{1-\frac{1}{\alpha}} y^{1-\frac{1}{\alpha}} m(d \alpha) .
$$

\section{Aggregated utilities from aggregating portfolios: the simplest case.}

In the previous section, we aggregated dual utilities $\widetilde{U}^{\alpha}$ under Pareto-optimality condition. We therefore obtained new results, in this stochastic utility framework. Nevertheless, Paretooptimality constrains the class because it imposes a common dual process. In the same way if we aggregate the utilities $U^{\alpha}$ then we should, by analogy, arrive at an equivalent constraint, i.e., we must have a unique optimal portfolio $X^{*}=X^{*, \alpha}, \forall \alpha$. To summarize, if we aggregate the utilities, the portfolio must be constrained and if we aggregate the duals then the state price is constrained. That limits our classes.

The goal in the sequel is to build an even larger class without any restrictions on wealths or state prices. Our idea is very simple but effective: the global wealth in an economy can only be the sum of the wealth of all investors. We then propose to aggregate wealth and state prices instead of utilities. In other words, we want to have freedom on the way to how we aggregate both portfolios and state prices.

To be more explicit, we must go beyond the abstract framework considered so far, we will place ourselves within the framework of an Itô financial market.

\subsection{The model setup:}

Let $W=\left(W_{1}, W_{2}, \ldots, W_{n}\right)^{T}\left({ }^{T}\right.$ for the transpose operator $)$ be a $n$-standard Brownian motion, defined on the filtered probability space $(\Omega, \mathcal{F}, \mathbb{P}) .\left(\mathcal{F}_{t}\right)_{t \geq 0}$ is the $\mathbb{P}$-augmented filtration generated by the Brownian motion $W$. Let $r$ denotes the market interest rate and $\eta$ denotes the market minimal risk premium. We assume that $r$ and $\eta$ are $\mathcal{F}$-progressively measurable satisfying the usual integrability condition

$$
\int_{0}^{T}\left(r_{t}+\left\|\eta_{t}\right\|^{2}\right) d t<+\infty \text { a.s } \forall T .
$$


It is standard to assume that any positive wealth process $X^{\kappa}$ follows a self-financing strategy $x \kappa$, that is a Itô-semimartingale satisfying the self-financing equation ${ }^{2}$

$$
d X_{t}^{\kappa}=X_{t}^{\kappa}\left[r_{t} d t+\kappa_{t} \cdot\left(d W_{t}+\eta_{t} d t\right)\right] .
$$

Throughout this paper, a process $X^{\kappa}$ solution of (4.2) is said to be an admissible test wealth if $\kappa_{t}$ is constrained to be in some linear space $\mathcal{R}_{t} \subset \mathbb{R}^{n}$, we denote by $\mathcal{R}_{t}^{\perp}$ its orthogonal. To fix the notations, the set of admissible wealth processes is denoted $\mathscr{X}^{+}$. We also recall that in this model setup $\mathscr{Y}$ is the set of positive semimartingales $Y^{\nu}$ whose dynamics are

$$
\frac{d Y_{t}^{\nu}}{Y_{t}^{\nu}}=-r_{t} d t+\left(\nu_{t}-\eta_{t}\right) \cdot d W_{t}, \quad \nu_{t} \in \mathcal{R}^{\perp}: \int_{0}^{T}\left\|\nu_{t}\right\|^{2} d t<\infty, \forall T .
$$

In other words, for any wealth process $\left(X^{\kappa}, \quad \kappa \in \mathcal{R}\right), Y^{\nu} X^{\kappa}$ is a local martingale. Since, $\nu . \eta=0$ for any $\nu \in \mathcal{R}^{\perp}, Y^{\nu}$ is decomposed as the product $Y^{0} \mathcal{E}(\nu)$ where $\mathcal{E}(\delta)$ denotes the exponential martingale defined by

$$
\mathcal{E}_{t}(\delta)=\exp \left(\int_{0}^{t} \delta_{s} \cdot d W_{s}-\frac{1}{2} \int_{0}^{t}\left\|\delta_{s}\right\|^{2} d s\right)
$$

Definition 4.1. To simplify notations, in what follows, a semimartingale $Z$ is said to satisfy a $S D E(\mu, \sigma)$ if it is solution of the equation

$$
d Z_{t}=\mu\left(t, Z_{t}\right) d t+\sigma\left(t, Z_{t}\right) d W_{t} .
$$

In this framework, dynamic utility $U$ is modeled as an Itô semimartingale following the dynamics

$$
d U(t, x)=\beta(t, x) d t+\gamma(t, x) d W_{t},
$$

and we recall, see El Karoui \& Mrad (2013), that regular stochastic utilities are solutions of the following HJB-SPDE,

$$
d U(t, x)=\left[-x r_{t} U_{x}(t, x)+\frac{1}{2 U_{x x}(t, x)}\left\|U_{x}(t, x) \eta_{t}+\gamma_{x}^{\mathcal{R}}(t, x)\right\|^{2}\right] d t+\gamma(t, x) \cdot d W_{t} .
$$

$\gamma$ denotes the volatility vector of $U$ and $\gamma_{x}^{\mathcal{R}}$ denotes the orthogonal projection onto the space of admissible portfolio $\mathcal{R}$ of its $x$-derivative denoted $\gamma_{x}$. Moreover, the optimal policy $\kappa^{*}$ is given by

$$
x \kappa_{t}^{*}(x)=-\frac{1}{U_{x x}(t, x)}\left(U_{x}(t, x) \eta_{t}+\gamma_{x}^{\mathcal{R}}(t, x)\right) .
$$

The associated optimal wealth process $X^{\kappa^{*}}$ is simply denoted $X^{*}$. The adjoint optimal process $Y_{t}^{*}=U_{x}\left(t, X_{t}^{*}\right)$ is solution of the $\operatorname{SDE}(4.4)$ with optimal dual policy given by $y \nu^{*}(t, y)=$ $\gamma_{x}^{\perp}\left(t,-\widetilde{U}_{y}(t, y)\right)$.

\footnotetext{
${ }^{2}$ In the case where the test wealth processes are not necessarily positive, the self-financing equation for a self-financing strategy $\pi$ is

$$
d X_{t}^{\pi}=X_{t}^{\pi} r_{t} d t+\pi_{t} \cdot\left(d W_{t}+\eta_{t} d t\right)
$$




\subsection{Randomized Risk Aversion}

The idea developed at the beginning of Section 4 is the starting point for the construction of a large class of consistent dynamic utilities. The proposed method will be first developed in details in the context of power utilities. This enables us, before describing the general method, to explain in a simple case different steps and the role of different parameters that are involved in the construction. The general case will be presented in Section 5. To get started, we recall a result giving sufficient conditions under which $\mathscr{X}^{+}$-consistent stochastic utilities are obtained by combining a power utility function $u^{\alpha}(x)=\frac{x^{1-\alpha}}{1-\alpha}$ with some positive process $Z$ satisfying

$$
\frac{d Z_{t}}{Z_{t}}=\mu_{t}^{Z} d t+\delta_{t}^{Z} \cdot d W_{t}
$$

where we assume in addition to (4.1), that $\int_{0}^{T}\left(\mu_{t}^{Z}+\left\|\delta_{t}^{Z}\right\|^{2}\right) d t<\infty$ a.s. $\forall T$.

For simplicity, for any random field $\left(M_{t}(z)\right)_{t, z}$ the process $\left(M_{t}(1)\right)_{t}$ (starting from 1 at $\left.t=0\right)$ is simply denoted by $M_{t}$.

In this semimartingale framework, we can give more details on the necessary dynamics of the process $Z^{\alpha}$ of Section 2.1 and also that of the optimal processes $X^{\alpha, *}$ and $Y^{\alpha, *}$. The following result is established in Mrad (2009).

Proposition 4.1. Let $u^{\alpha}$ be a power utility with risk aversion $\alpha$ that is $u^{\alpha}(x)=\frac{x^{1-\alpha}}{1-\alpha}$. Assume that parameters of diffusion $\mu^{Z}, \delta^{Z}$ of $Z$ satisfy the following equation

$$
\mu_{t}^{Z}=-(1-\alpha) r_{t}-\frac{1-\alpha}{2 \alpha}\left\|\eta_{t}+\delta_{t}^{Z, \sigma}\right\|^{2}
$$

Then the stochastic process $U^{\alpha}$ defined by $U^{\alpha}(t, x)=Z_{t} u^{\alpha}(x)$ is a $\mathscr{X}^{+}$-consistent utility with optimal policy

$$
\kappa_{t}^{*, \alpha}(x)=\frac{1}{\alpha}\left(\eta_{t}+\delta_{t}^{Z, \sigma}\right) .
$$

In turn, the optimal wealth $X^{\alpha, *}$ and dual $Y^{\alpha, *}$ processes are given by,

$$
\left\{\begin{aligned}
X_{t}^{*, \alpha}(x): & =x \bar{X}_{t}^{*, \alpha}=x e^{\int_{0}^{t}\left(r_{s}+\frac{1}{\alpha}\left(\eta_{s}+\delta_{s}^{Z, \sigma}\right) \cdot \eta_{s}\right) d s} \mathcal{E}_{t}\left(\frac{\eta+\delta^{Z, \sigma}}{\alpha}\right) \\
Y_{t}^{\alpha, *}(y): & =U_{x}^{\alpha}\left(t, X_{t}^{*}\left(y^{-\frac{1}{\alpha}}\right)\right)=y \bar{Y}_{t}^{\delta^{Z, \perp}}=y e^{-\int_{0}^{t} r_{s} d_{s}} \mathcal{E}_{t}\left(\delta^{Z, \perp}-\eta\right)
\end{aligned}\right.
$$

Remark 4.1. (i) $X^{*, \alpha}$ and $Y^{*, \alpha}$ are linear with respect to their initial conditions. This property will play an important role in the sequel. In particular, it provides an explicit formula for the corresponding inverse flows.

(ii) $Y^{*, \alpha}(y)=y \bar{Y}^{\delta^{Z, \perp}}$ is the same for all $\alpha$.

(iii) The volatility $\delta^{Z}$ can be chosen independently of $\alpha$ and thus $\alpha \kappa^{*, \alpha}=\alpha^{\prime} \kappa^{*, \alpha^{\prime}}$. 
Random risk aversion: At this stage the coefficient $\alpha$, which is the relative risk aversion, was supposed constant, it is about the simplest case of the power $\mathscr{X}^{+}$-consistent utilities considered in Section 2.1. But it is completely conceivable that this risk aversion is in general random. Indeed we can imagine at date $t=0$ that the investor pulls at random the value of this coefficient. For every value $\alpha$ he associates:

(i) a weight $m(\alpha)$ ( $m$ is a finite positive measure s.t $\left.\int_{\mathbb{R}_{+}^{*}} m(d \alpha)=1\right)$,

(ii) a proportion $x_{\alpha}(x)$ of its initial wealth (strictly increasing on $x, x_{\alpha}(x) \rightarrow \infty$ if $x \rightarrow \infty$ and $\left.x_{\alpha}(0)=0\right)$ that he is going to invest on the financial market by considering the utility process $U^{\alpha}$. He will so realize a wealth $X^{*, \alpha}\left(x_{\alpha}(x)\right)$ associated with this edition and achieved by the optimal strategy $\kappa^{\alpha, *}$.

His final wealth is consequently the sum of the processes $X^{*, \alpha}\left(x_{\alpha}(x)\right)$ weighted by the measure $m$, i.e., using the notation $\bar{X}_{t}^{*, \alpha}:=X_{t}^{*, \alpha}(1)$

$$
\left\{\begin{array}{l}
X_{t}^{*}(x)=\int_{\mathbb{R}_{+}^{*}} X_{t}^{*, \alpha}\left(x_{\alpha}(x)\right) m(d \alpha)=\int_{\mathbb{R}_{+}^{*}} x_{\alpha}(x) \bar{X}_{t}^{*, \alpha} m(d \alpha), \\
X_{0}^{*}(x)=x=\int_{\mathbb{R}_{+}^{*}} x_{\alpha}(x) m(d \alpha) .
\end{array}\right.
$$

By monotonicity assumption of $x_{\alpha}, X^{*}$ is strictly increasing on $x$ and satisfies

$$
\frac{d X_{t}^{*}(x)}{X_{t}^{*}(x)}=r_{t} d t+\kappa_{t}^{*}\left(X_{t}^{*}(x)\right) \cdot\left(d W_{t}+\eta_{t} d t\right)
$$

where the volatility vector $\kappa_{t}^{*}\left(X_{t}^{*}(x)\right)$ is given, since $\kappa_{t}^{*, \alpha}(x)=\frac{1}{\alpha}\left(\eta_{t}+\delta_{t}^{Z, \sigma}\right)$, by

$$
\kappa_{t}^{*}\left(X_{t}^{*}(x)\right):=\left[\int_{\mathbb{R}_{+}^{*}} \frac{X_{t}^{*, \alpha}\left(x_{\alpha}(x)\right)}{X_{t}^{*}(x)} \frac{1}{\alpha} m(d \alpha)\right]\left(\eta_{t}+\delta_{t}^{Z, \sigma}\right) .
$$

Denote by $\mathcal{X}(x)$ the inverse function of $X^{*}(x)$ and let $u$ be any utility function of class $\mathcal{C}^{1}(] 0,+\infty[)$ continuous at $x=0$ with $u(0)=0$. Furthermore, in addition to (4.1), assume for the rest of this section the following,

Assumption 4.1. Suppose that $\alpha \mapsto \frac{\delta^{Z}}{\alpha}$ is uniformly bounded.

First, this assumption ensures that the process $X^{*} Y_{t}^{\delta^{Z, \perp} 3}$ is a martingale (strong orthogonality in Theorem 2.1, Remark 2.1). Taking $Y_{t}^{*}(y)=y \bar{Y}_{t}^{\delta^{Z, \perp}}\left(\bar{Y}_{t}^{\delta^{Z, \perp}}:=Y_{t}^{\delta^{Z, \perp}}(1)\right)$, it follows, according to Theorem 2.1 that the process $U(t, x)$ defined by

$$
U(t, x)=\bar{Y}_{t}^{\delta^{Z, \perp}} \int_{0}^{x} u_{x}(\mathcal{X}(t, z)) d z
$$

is a consistent stochastic utility.

The following result summarizes our first construction from aggregating portfolio without Pareto-optimality.

\footnotetext{
${ }^{3}$ Where we recall that $Y^{\delta^{Z, \perp}}$ is the process defined by $\frac{d Y_{t}^{\delta^{Z, \perp}}}{Y_{t}^{\delta^{Z, \perp}}}=-r_{t} d t+\left(\delta_{t}^{Z, \perp}-\eta_{t}\right) \cdot d W_{t}$ and $\delta^{Z, \perp}$ is the orthogonal projection of $\delta$ onto the space $\mathcal{R}^{\perp}$.
} 
Theorem 4.1. Let $m$ be a Borel measure and $\left(x_{\alpha}(.)\right)_{\alpha \in \mathbb{R}}$ be a class of increasing functions s.t. $x=\int_{\mathbb{R}_{+}^{*}} x_{\alpha}(x) m(d \alpha)$. Consider the state price $Y^{*}=y Y^{\delta^{Z, \perp}}$ and $\left(X^{*, \alpha}\right)_{\alpha \in \mathbb{R}}$ the family of optimal processes in Proposition 4.1 and define $X^{*}$ by

$$
X_{t}^{*}(x)=\int_{\mathbb{R}_{+}^{*}} X_{t}^{*, \alpha}\left(x_{\alpha}(x)\right) m(d \alpha) .
$$

It is a strictly increasing admissible wealth process solution of (4.15). Under Assumption 4.1, for any initial utility function $u$, the progressive utility

$$
U(t, x)=\bar{Y}_{t}^{\delta^{Z, \perp}} \int_{0}^{x} u_{x}(\mathcal{X}(t, z)) d z
$$

is a non-zero volatility $\mathscr{X}$-consistent stochastic utility with optimal pair $\left(X^{*}(x), Y^{*}\right)$. Furthermore, the convex conjugate of $U$ is given by

$$
\widetilde{U}(t, y)=\int_{y}^{\infty} X_{t}^{*}\left(-\tilde{u}_{y}\left(\frac{z}{\bar{Y}_{t}^{\delta^{Z, \perp}}}\right)\right) d z=\int_{y}^{\infty} \int_{\mathbb{R}_{+}^{*}} x_{\alpha}\left(-\tilde{u}_{y}\left(\frac{z}{\bar{Y}_{t}^{\delta^{Z, \perp}}}\right)\right) X_{t}^{*, \alpha} m(d \alpha) d z .
$$

In that way, we generate a family of non-standard consistent utilities from aggregating optimal wealth processes $X^{*, \alpha}$ associated with a family of power consistent utilities. Note that, this stochastic utilities depend, first on the family of functions $x_{\alpha}, \alpha \in \mathbb{R}_{+}^{*}$, second on the choice of the measure $m$, third on the process $Z\left(\mu^{Z}, \delta^{Z}\right)$ and finally on the initial condition $u$. Note also, that the initial utility function $u$ is not necessarily a power one. Moreover, this stochastic utilities are built from optimal wealth and dual processes associated with simple utilities indexed by a parameter $\alpha$.

Example Let $u$ in (4.20) such that its Fenchel's transform function $\tilde{u}$ is given by

$$
-\tilde{u}_{y}(y)=\left(u_{x}\right)^{-1}(y)=\int_{\mathbb{R}_{+}^{*}} y^{-\frac{1}{\alpha}} m(d \alpha)
$$

Take $x_{\alpha}=\left[\left(u_{x}\right)^{-1}\right]^{-\frac{1}{\alpha}}$ that is $x_{\alpha}\left(u_{x}\right)(x)=x^{-\frac{1}{\alpha}}$. Take also, $r \equiv 0$ and $\delta \equiv 0$ (i.e., $Y^{\delta^{\perp}}=Y^{0}$ is the inverse of the market numeraire portfolio corresponding to $\nu=0$ in (4.4)). Subsequently, the dual is given by

$$
\widetilde{U}(t, y)=\int_{\mathbb{R}_{+}^{*}} \frac{1}{1-\frac{1}{\alpha}}\left(1-y^{1-\frac{1}{\alpha}} \bar{X}_{t}^{*, \alpha}\left(Y_{t}^{0}\right)^{\frac{1}{\alpha}}\right) m(d \alpha) .
$$

It suffices to observe that

$$
X_{t}^{*, \alpha}\left(Y_{t}^{0}\right)^{\frac{1}{\alpha}}=\exp \left(-\frac{1-\alpha}{2 \alpha} \int_{0}^{t}\left\|\eta_{s}\right\|^{2} d s\right)
$$

and then to conclude with

$$
\widetilde{U}(t, y)=\int_{\mathbb{R}_{+}^{*}} \frac{1}{1-\frac{1}{\alpha}}\left(1-y^{1-\frac{1}{\alpha}} e^{-\frac{1-\alpha}{2 \alpha} \int_{0}^{t}\left\|\eta_{s}\right\|^{2} d s}\right) m(d \alpha)
$$


which is the convex conjugate of the time-decreasing consistent (forward) utilities characterized by Berrier et al. (2009) and Musiela \& Zariphopoulou (2010, 2011). Certainly this family of utilities is a special case of the class that we propose in this section, but it should be emphasized that the nice characterization obtained by the authors cited above is not obvious because the starting point for their studies is the search for convex solutions of some space-time harmonic PDE. The following paragraph recalls their results but also offers a simpler new proof (in the same spirit of this paper) explaining how these time-decreasing utilities are necessarily mixtures of power utilities.

\subsection{Decreasing consistent utilities: new interpretation and a direct characterization}

Herein, we deepen the class of decreasing (in time) consistent utilities. They have a volatility vector $\gamma$ identically zero which simplifies considerably the dual SPDE. Indeed, taking $\gamma \equiv 0$ and $r \equiv 0$, it follows from equation (4.8), that $U$ is a solution of the following PDE

$$
d U(t, x)=\frac{1}{2} \frac{U_{x}(t, x)^{2}}{U_{x x}(t, x)}\left\|\eta_{t}\right\|^{2} d t
$$

whereas the convex conjugate $\widetilde{U}$ satisfies

$$
\widetilde{U}_{t}(t, y)(\omega)=-\frac{1}{2} y^{2} \widetilde{U}_{y y}(t, y)(\omega)\left\|\eta_{t}(\omega)\right\|^{2}
$$

which implies, by convexity, that $t \mapsto \widetilde{U}(t, y)$ is a decreasing function. Moreover, it is easy to recognize that the right hand side of this PDE is the diffusion's operator $L_{t, y}^{G B}(\omega)$ of a geometric Brownian motion with coefficients $\eta_{t}(\omega)$ applied to $\widetilde{U}: \widetilde{U}_{t}(t, y)(\omega)=-L_{t, y}^{G B} \widetilde{U}(t, y)(\omega)$. This observation suggests to look for positive solutions which are space-time harmonic functions of a geometric Brownian motion. Using the results of Widder (1963, 1975), Berrier et al. (2009) and Musiela \& Zariphopoulou (2010, 2011) show the following result which characterizes all regular dual convex conjugate of decreasing consistent utilities.

Theorem 4.2. Let $U(t, x)$ be a regular random field of class $\mathcal{C}^{1} \times \mathcal{C}^{3}$ on $(t, x)$. Assume that $U$ satisfies the PDE (4.25). Then $U$ is a consistent stochastic utility if and only if there exists a constant $C \in \mathbb{R}$ and a finite Borel measure $m$, supported on the interval $(0,+\infty)$ with everywhere finite Laplace transform, such that

$$
\left\{\begin{aligned}
\widetilde{U}(t, y) & =\int_{\mathbb{R}_{+}^{*}} \frac{1}{1-\frac{1}{\alpha}}\left(1-y^{1-\frac{1}{\alpha}} e^{-\frac{1-\alpha}{2 \alpha} \int_{0}^{t}\left\|\eta_{s}\right\|^{2} d s}\right) m(d \alpha)+C, \\
\widetilde{U}_{y}(0, y) & =-\int_{\mathbb{R}_{+}^{*}} y^{-\frac{1}{\alpha}} m(d \alpha) .
\end{aligned}\right.
$$

Moreover the optimal wealth process is strictly increasing and regular with respect to its initial condition $x$. 
At date $t=0$ the dual conjugate $\widetilde{U}(0, y)$ is the integral of the the convex conjugate $\frac{1-y^{1-\frac{1}{\alpha}}}{1-\frac{1}{\alpha}}$ of power utilities weighted by the measure $m$. As in our construction in Section 4.2, the investor starts from a power utility for which he pull at random the risk aversion; for any realization $\alpha$ he associates the power utility $u^{\alpha}$ weighted by $m(d \alpha)$. The derivative of the convex conjugate of his utility at any date $t$ is then the integral of the convex conjugates of power utility where the deterministic measure $m$ becomes stochastic $m_{t}(d \alpha):=e^{-\frac{1-\alpha}{2 \alpha} \int_{0}^{t}\left\|\eta_{s}\right\|^{2} d s} m(d \alpha)$ to ensure that the process $\widetilde{U}$ is the dual of a decreasing consistent utility. From this last point, one can also see that these utilities are a particular case of Section 3 where we aggregate any dual utilities. Thus applying Theorem 3.1, we obtain a direct characterization of the decreasing consistent utility as a sup-convolution.

Theorem 4.3 (New Characterization). According to Theorem 3.1, any decreasing forward utility $U$ is a Sup-Convolution:

$$
U(t, x)=\sup _{x_{\alpha}: \int x_{\alpha}(x) m(d \alpha)=x} \int \frac{\left(x_{\alpha}(x)\right)^{1-\alpha}}{1-\alpha} e^{\frac{1-\alpha}{2} \int_{0}^{t}\left\|\eta_{s}\right\|^{2} d s} m(d \alpha) .
$$

Alternative proof of Theorem 4.2, using Mellin's Transform The fact that the dual utility of a time-decreasing progressive utility is not at all surprising and quite natural since the aggregation of several solutions of a linear PDE remains a solution of the same equation. Herein, we give a new proof of Theorem 4.2, different from that given Berrier et al. (2009) and Musiela \& Zariphopoulou (2010, 2011) where authors use the Widder's Theorem. The advantage of this new proof is its simplicity, does not require any technicality and knowledge in PDE's theory, it is a nice illustration of aggregations which are the subject of this paper. It is based on Mellin's transform and exploits the linearity of the dual equation (4.26).

To do this, we need the the following integrability hypothesis.

Assumption 4.2. The initial condition $u$ is such that there exists a positive finite Borel measure $\mu$ supported on $\mathbb{R}$ such that $y \mapsto \tilde{u}(y) y^{p}$ is integrable with respect to $\mu$ for any $p \in \mathbb{R}$ $\left.\left(\int_{0}^{\infty} \tilde{u}(y) y^{p} \mu(d y)\right)<+\infty\right)$.

A simple example of such functions $\tilde{u}$ satisfying this integrability condition, is the class of $\tilde{u}$ that are bounded by a power function, that is there exist $p_{0} \in \mathbb{R}$ and $c>0$ such that $|\tilde{u}(y)| \leq c y^{-p_{0}}, y>0$ (these type of functions $\tilde{u}$ have been considered in Karatzas \& Shreve (2001)). In this case, one can easily observes that $y \mapsto \tilde{u}(y) y^{p} \leq y^{p-p_{0}}$ is integrable with respect to any measure $\nu$ supported on $] 0, \infty\left[\right.$ for any $p<p_{0}-1$.

New proof of Theorem 4.2 using Mellin's Transform. Clearly the dual equation (4.26) is simpler to study than the primal one (4.25). So we will investigate the problem in terms of 
the dual convex. Indeed, by martingale property of $\widetilde{U}\left(t, y Y_{t}^{0}\right)$, one can easily observe, using Itô-Ventzel's formula, that

$$
d \widetilde{U}\left(t, y Y_{t}^{0}\right)=-y Y_{t}^{0} \widetilde{U}_{y}\left(t, y Y_{t}^{0}\right) \eta_{t} d W_{t}
$$

which becomes, denoting by $\widetilde{V}(t, y):=\widetilde{U}\left(t, y Y_{t}^{0}\right)$,

$$
d \widetilde{V}(t, y)=-y \widetilde{V}_{y}(t, y) \eta_{t} d W_{t}
$$

Let now introduce Mellin's transform which is an integral transform that may be regarded as the multiplicative version of the bilateral Laplace transform. Mellin's transform of $\widetilde{V}$ is defined by

$$
M_{t}(p):=\int_{0}^{+\infty} y^{p-1} \widetilde{V}(t, y) \mu(d y)
$$

Since the process $\widetilde{U}\left(t, y Y_{t}^{0}\right)$ is a martingale, $M_{t}(p)$ is well defined, finite almost surely. Indeed

$$
\mathbb{E}\left(M_{t}(p)\right) \leq \int_{0}^{+\infty} y^{p-1} \tilde{u}(y) \mu(d y)<+\infty .
$$

Let us now focus on the dynamics of the process $M$. For this, remark that Mellin's transform $\hat{M}$ of $y \widetilde{V}_{y}(t, y)$, using integration by part and integrability conditions, is given by

$$
\begin{aligned}
\hat{M}_{t}(p): & =\int_{0}^{+\infty} y^{p} \widetilde{V}_{y}(t, y) \mu(d y)=-p \int_{0}^{+\infty} y^{p} \widetilde{V}(t, y) \mu(d y)+C(p) \\
& =-p M_{t}(p)+C(p)
\end{aligned}
$$

with $C(p):=\lim _{y \rightarrow 0} y^{p} \widetilde{V}(t, y)$ which is null except for at most a single point $p_{0}$. So without loss of generality, we assume $C(p)=0$ everywhere. This implies that the process $M_{t}(p)$ is solution of

$$
d M_{t}(p)=p M_{t}(p) \eta_{t} \cdot d W_{t}
$$

which has a unique solution given by

$$
M_{t}(p)=M_{0}(p) \mathcal{E}\left(p \int_{0}^{t} \eta_{s} \cdot d W_{s}\right),
$$

which easily rewrites

$$
M_{t}(p)=M_{0}(p)\left(Y_{t}^{0}\right)^{-p} e^{-p \frac{p+1}{2} \int_{0}^{t}\left\|\eta_{s}\right\|^{2} d s}
$$

To come back to $\widetilde{V}(t, y)=\widetilde{U}\left(t, y Y_{t}^{0}\right)$, we apply Mellin's inverse transform: there exists a constant $C_{1}$ such that

$$
\widetilde{U}\left(t, y Y_{t}^{0}\right)=\int_{-\infty}^{+\infty} y^{-p} M_{t}(p) \mu(d p)+C_{1}
$$


which becomes, using (4.36),

$$
\widetilde{U}\left(t, y Y_{t}^{0}\right)=\int_{-\infty}^{+\infty}\left(y Y_{t}^{0}\right)^{-p} e^{-p \frac{p+1}{2} \int_{0}^{t}\left\|\eta_{s}\right\|^{2} d s} M_{0}(p) \mu(d p)+C_{1}
$$

and finally, by change of variable $y Y_{t}^{0} \mapsto y$, it follows

$$
\widetilde{U}(t, y)=\int_{-\infty}^{+\infty} y^{-p} e^{-\frac{p(1+p)}{2} \int_{0}^{t}\left\|\eta_{s}\right\|^{2} d s} M_{0}(p) \mu(d p)+C_{1} .
$$

Moreover, note that the condition $\widetilde{U}_{t}<0$ holds if and only if $p>-1$. Thus, this leads to

$$
\widetilde{U}(t, y)=\int_{-1}^{+\infty} y^{-p} e^{-\frac{p(1+p)}{2} \int_{0}^{t}\left\|\eta_{s}\right\|^{2} d s} M_{0}(p) \mu(d p)+C_{1}
$$

and by change of variable $q=p+1$

$$
\widetilde{U}(t, y)=-\int_{0}^{+\infty} \frac{1}{1-q} y^{1-q} e^{-\frac{q(1-q)}{2} \int_{0}^{t}\left\|\eta_{s}\right\|^{2} d s}(1-q) M_{0}(q-1) \mu(d q)+C_{1} .
$$

Denote by $\nu$ the finite Borel measure supported on $\left[0, \infty\left[\right.\right.$ defined by $\nu(d p):=M_{0}(p) \mu(d p) / p$, we get

$$
\widetilde{U}(t, y)=\int_{0}^{+\infty} \frac{1}{1-q}\left(1-y^{1-q} e^{-\frac{q(1-q)}{2} \int_{0}^{t}\left\|\eta_{s}\right\|^{2} d s}\right) \nu(d q)+C
$$

for some constant $C$. In particular, we have that the initial dual utility $\tilde{u}$ is necessarily of the form

$$
\tilde{u}(y)=\int_{0}^{+\infty} \frac{1}{1-q}\left(1-y^{1-q}\right) \nu(d q)+C .
$$

Which is the characterization of a decreasing consistent utility Theorem 4.2.

Note that the optimal wealth process $X^{*}$ is given by the closed formula

$$
X^{*}(t, x)=\int_{0}^{\infty}\left(u_{x}(x) Y_{t}^{0}\right)^{-p} e^{\frac{-p(1-p)}{2} \int_{0}^{t}\left\|\eta_{s}\right\|^{2} d s} \nu(d p)
$$

and it is strictly increasing with respect to its initial condition $x$. Moreover, the optimal dual process is $Y^{*}(y)=y Y^{0}$ with inverse $\mathcal{Y}(y):=\left(Y^{*}\right)^{-1}(y)=y / Y^{0}$. Then, one can easily verifies that the random field $\widetilde{U}_{y}$ is obtained by the general form of Theorem 2.1, that is $\widetilde{U}_{y}(t, y)=-X^{*}\left(t,-\tilde{u}_{y}(\mathcal{Y}(t, y))\right)$.

Of course, this result was already established in Berrier et al. (2009) and Musiela \& Zariphopoulou (2010) but the novelty here, that merits to be integrated into this work, is that we propose a very simple and direct proof of the result, without using the time change techniques and Widder's results. Besides, Musiela \& Zariphopoulou (2010, 2011) developed several examples with different measures $m$ as well as properties of the associated optimal wealth, something we do not develop here. 
Comments Before continuing our investigations, the following point needs to be highlighted. In a first reading one might think that the necessary condition (4.43) on the initial function $u$ and the dependence (4.44) of the optimal wealth on this function is in contradiction with the rest of the paper where we emphasize that the choice of $u$ does not play a very important role in our main results. But in fact, this result is entirely consistent with the previous paragraphs and even a nice application of our results. Indeed, starting from the optimal portfolio $X^{*}$ given by equation (4.44), one can easily construct a new consistent utility having $X^{*}$ as optimal process starting from an initial utility function $v$ and generating a monotonic optimal dual process $Y^{\nu^{*}}(y)$ (not necessarily equal to $y Y^{0}$ ) with inverse $\mathcal{Y}$, as follows:

$$
\begin{aligned}
\widetilde{V}_{y}(t, y) & =-X^{*}\left(t,-\tilde{v}_{y}(\mathcal{Y}(t, y))\right) \\
& =-\int_{0}^{\infty}\left(u_{x}\left(-\tilde{v}_{y}(\mathcal{Y}(t, y))\right) Y_{t}^{0}\right)^{-p} e^{\frac{-p(1-p)}{2} \int_{0}^{t}\left\|\eta_{s}\right\|^{2} d s} \nu(d p)
\end{aligned}
$$

Clearly this process is strictly convex non-zero volatility (not decreasing in time). Moreover $\widetilde{V}$ is the Fenchel transform of a consistent utility $V$. To be convinced, just apply the results of Theorem 2.7 in El Karoui \& Mrad (2013), to the compound process $X^{*}\left(t,-\tilde{v}_{y}(\mathcal{Y}(t, y))\right)$, using the fact that $\mathcal{Y}$ is a solution of a SPDE since it is the inverse process of $Y^{*}$ solution of a SDE.

Finally, if we want $V$ to be a time-decreasing utility that is if we want its volatility vector is null, then a simple application of Itô's formula leads to $\mathcal{Y}(t, y)=y / Y_{t}^{0}, u_{x}\left(-\tilde{v}_{y}(\mathcal{Y}(t, y)) Y_{t}^{0}\right)=y$ which consistent with Theorem 4.2.

\section{$5 \quad$ Aggregating wealths and state density price processes}

In this section, we go even further in our reasoning and consider couples $\left(X^{*, \alpha}, Y^{*, \alpha}\right)$ associated with any dynamic utilities $\left(U^{\alpha}\right)_{\alpha \in \mathbb{R}}$ without any restrictions either on the type or on the meaning of the parameter $\alpha$, with the possibility of taking $X^{*, \alpha}$ not necessarily positive (with a little more integrability conditions). This does not change our construction in any way because we do not really use $U^{\alpha}$ to built a new utility. The important point is that $\left(X^{*, \alpha}, Y^{*, \alpha}\right)$ are monotonic in their initial conditions, if this is the case then the aggregation pair $\left(X^{*}, Y^{*}\right)$ are still monotonic and one can apply Theorem 2.1 to generate a new consistent dynamic utilities.

Thus, this section generalizes the method proposed and provides a larger class of consistent utilities which will allow us to consider a much more heterogeneous agents. The power and exponential utilities in what follows are also concrete examples to illustrate the proposed method. 


\subsection{Randomized parameter $\alpha$, the general principle:}

The idea developed in Section 4.2 is generalized as follows.

At date $t=0$ the investor pulls at random the value of the risk aversion coefficient ${ }^{4}$. For every value $\alpha$ he associates:

(i) a weight $m(\alpha)$ ( $m$ is a finite positive measure s.t $\left.\int_{\mathbb{R}_{+}^{*}} m(d \alpha)=1\right)$,

(ii) a proportion $x_{\alpha}(x)$ of its initial wealth (positive strictly increasing on $x$ ) that he is going to invest on the financial market by considering $U^{\alpha}$ as utility, he will so realize $X^{*, \alpha}\left(x_{\alpha}(x)\right)$ as wealth achieved by the optimal policy $\pi^{\alpha, * 5}$.

(iii) a function $y_{\alpha}(),. \alpha \in \mathbb{R}_{+}^{*}$ (strictly increasing $y, y_{\alpha}(y) \rightarrow \infty$ if $y \rightarrow \infty$ and null for $y=0$ ).

His final (global) wealth is consequently the sum of the processes $X^{*, \alpha}\left(x_{\alpha}(x)\right)$ weighted by the measure $m$, i.e.

$$
X_{t}^{*}(x)=\int_{\mathbb{R}_{+}^{*}} X_{t}^{*, \alpha}\left(x_{\alpha}(x)\right) m(d \alpha), \quad X_{0}^{*}(x)=x=\int_{\mathbb{R}_{+}^{*}} x_{\alpha}(x) m(d \alpha) .
$$

By monotonicity of $x_{\alpha}$ and that of $X^{*, \alpha}, X^{*}$ is strictly increasing on $x$ and satisfies

$$
d X_{t}^{*}(x)=r_{t} X_{t}^{*}(x) d t+\pi_{t}^{*}\left(X_{t}^{*}(x)\right) \cdot\left(d W_{t}+\eta_{t} d t\right)
$$

where the volatility vector $\kappa_{t}^{*}\left(X_{t}^{*}(x)\right)$ is given by

$$
\pi_{t}^{*}\left(X_{t}^{*}(x)\right):=\int_{\mathbb{R}_{+}^{*}} \pi_{t}^{\alpha, *}\left(X_{t}^{*, \alpha}\left(x_{\alpha}(x)\right)\right) m(d \alpha) .
$$

By analogy, we consider the state price density $Y^{*}$ defined as the sum of the processes $Y^{\alpha, *}$ weighted by the measure $m$, i.e.

$$
Y_{t}^{*}(y)=\int_{\mathbb{R}_{+}^{*}} Y_{t}^{*, \alpha}\left(y_{\alpha}(y)\right) m(d \alpha), \quad Y_{0}^{*}(y)=y \stackrel{\text { def }}{=} \int_{\mathbb{R}_{+}^{*}} u_{x}^{\alpha}\left(y_{\alpha}(y)\right) m(d \alpha) .
$$

Consequently, the increasing process $Y_{t}^{*}$ solves

$$
\frac{d Y_{t}^{*}(y)}{Y_{t}^{*}(y)}=-r_{t} d t+\left(\nu_{t}^{*}\left(Y_{t}^{*}(y)\right)-\eta_{t}\right) d W_{t}
$$

with

$$
\nu_{t}^{*}\left(Y_{t}^{*}(y)\right):=\int_{\mathbb{R}_{+}^{*}} \frac{Y_{t}^{\alpha, *}\left(y_{\alpha}(y)\right)}{\int_{\mathbb{R}_{+}^{*}} Y_{t}^{\alpha, *}\left(y_{\alpha}(y)\right) m(d \alpha)} \nu_{t}^{\alpha, *}\left(Y_{t}^{\alpha, *}\right) m(d \alpha) .
$$

Subsequently, assuming $\alpha \mapsto \pi^{*, \alpha}$ and $\alpha \mapsto \nu^{*, \alpha}$ are uniformly bounded by some $t$-integrable function, it follows that, the optimal policies satisfy

$$
\int_{0}^{T}\left(\left\|\pi_{s}^{*}\left(X_{s}^{*}(x)\right)\right\|^{2}+\left\|\nu_{s}^{*}\left(Y_{s}^{*}(y)\right)\right\|^{2}\right) d s<\infty, \text { a.s. } \forall T .
$$

\footnotetext{
${ }^{4}$ To be more precise, if $u^{\alpha}$ is a power utility $\alpha$ is the relative risk aversion and the absolute risk aversion if $u^{\alpha}$ is an exponential one.

${ }^{5} \pi^{\alpha, *}(x)=x \kappa^{*}$ when wealth processes are modeled positive.
} 
Thus, the pair of processes $\left(X_{t}^{*}(x), Y_{t}^{*}(y)\right)$ which are increasing with respect to their initial condition $(x, y)$ are such that their product $X^{*}(x) Y^{*}(y)$ is a true martingale for any $(x, y)$ (Strong orthogonality in Theorem 2.1, Remark 2.1).

Then, according to Theorem 2.1, the process $U$ defined by

$$
U(t, x)=\int_{0}^{x} Y^{*}\left(t, u_{x}(\mathcal{X}(t, z))\right) d z
$$

is a $\mathscr{X}$-consistent stochastic utility. The associated optimal portfolio is $X^{*}$ and the optimal state price density process is $Y^{*}$, in particular $Y_{t}^{*}\left(u_{x}(x)\right)=U_{x}\left(t, X_{t}^{*}(x)\right)$.

To summarize, we need the following global Assumption,

Assumption 5.1. (i) Let $\left(X^{*, \alpha}\right)_{\alpha \in \mathbb{R}}$ be a family of positive admissible wealth processes solving $\left(S D E\left(x r+\pi^{*, \alpha} \eta, \pi^{*, \alpha}\right)\right)_{\alpha \in \mathbb{R}}$ and assume that $x \mapsto X_{t}^{*, \alpha}(x)$ are a.s. strictly increasing for any $t$ from $\mathbb{R}$ to $\mathbb{R}$.

(ii) Let $\left(Y^{*, \alpha}\right)_{\alpha \in \mathbb{R}}$ be a family of state price density processes solving $\left(S D E\left(-y r, y\left(\nu^{*, \alpha}-\right.\right.\right.$ $\eta))_{\alpha \in \mathbb{R}^{*}}$. Assume and $y \mapsto Y_{t}^{*, \alpha}(y)$ to be a.s. strictly increasing for any $t$ from $\mathbb{R}^{+}$to $\mathbb{R}^{+}$.

(iii) $m$ is a positive Borel measure and $\left(x_{\alpha}\right)_{\alpha \in \mathbb{R}}$ and $\left(y_{\alpha}\right)_{\alpha \in \mathbb{R}}$ are two families of increasing functions s.t. $x=\int_{\mathbb{R}_{+}^{*}} x_{\alpha}(x) m(d \alpha), \quad y=\int_{\mathbb{R}_{+}^{*}} y_{\alpha}(y) m(d \alpha)$ for any $x, y$.

Under this Assumption, the following result summarizes our general construction from aggregating both portfolios and state pricess without Pareto-optimality.

Theorem 5.1. Under Assumption 5.1, the following aggregations,

$$
\begin{cases}X_{t}^{*}(x):=\int_{\mathbb{R}_{+}^{*}} X_{t}^{*, \alpha}\left(x_{\alpha}(x)\right) m(d \alpha), \quad X_{0}^{*}(x)=x \\ Y_{t}^{*}(y)=\int_{\mathbb{R}_{+}^{*}} Y_{t}^{*, \alpha}\left(y_{\alpha}(y)\right) m(d \alpha), \quad Y_{0}^{*}(y)=y\end{cases}
$$

are respectively an admissible wealth process $x$-increasing, solution of the $S D E\left(x r+\pi^{*} \eta, x \pi^{*}\right)$ and a price density process $y$-increasing, solution of the $S D E\left(-y r, y\left(\nu^{*}-\eta\right)\right)$, where $\pi^{*}$ is given by (5.3) and $\nu^{*}$ is given by (5.6).

Moreover, if (5.7) holds and $X^{*, \alpha}(x) Y^{*, \alpha^{\prime}}(y)$ are martingales for any $x, y, \alpha$ and $\alpha^{\prime}$, then for any initial utility function $u$, the progressive utility $U$ defined by

$$
U(t, x)=\int_{0}^{x} Y_{t}^{*}\left(u_{x}(\mathcal{X}(t, z))\right) d z \text { where } \mathcal{X}(x)=\left(X^{*}\right)^{-1}(x),
$$

is $\mathscr{X}$-consistent with optimal processes $X^{*}$ and $Y^{*}$.

Remark 5.1. It is important to note that this section generalizes not only the results of Section 4.2 but also that of Section 3 with Sup-convolution and Pareto-optimality, it suffices to impose the same state price and to choose well the initial allocation functions $x_{\alpha}$. 
The construction described in this section is very general, nonetheless to provide illustrative examples, it is essential to give an explicit formula for the inverse flow $\mathcal{X}$. We can always invert $X^{*}$ numerically and simulate $U$, see Section 4 of Gobet \& Mrad (2018), but it is almost impossible to give a closed formula for $\mathcal{X}$ in general except for a few special cases. One of these special cases is the framework of the Proposition 4.1 and its extension Proposition 5.1 below. The proposed class of new dynamic utilities is very rich.

\section{$5.2 \quad$ Examples}

In the construction proposed in Section 4.2, which we are going to extend here, the volatility $\delta^{Z}$ of the process $Z$ (used to define dynamic utility from deterministic one in Proposition 4.1 ) is independent of the index parameter $\alpha$, while the investor can decide to assign to each function $u^{\alpha}$ a process $Z^{(\alpha)}$ whose volatility $\delta^{(\alpha)}$ depends on the risk aversion $\alpha$. Moreover, for simplicity we have chosen the process $Y^{*}(y)$ proportional to $\bar{Y}^{\delta^{(\alpha), \perp}}:=Y^{\delta^{(\alpha), \perp}}(1)$, i.e. $Y^{*}(y)=y \bar{Y}^{\delta^{(\alpha), \perp}}$, so it is possible, as explained above, to construct by the same reasoning, as the one used to built a global wealth $X^{*}$, a state price density process $Y^{*}$ using a family of functions $\left\{y_{\alpha}, \alpha \in \mathbb{R}_{+}^{*}\right\}$. All these observations with the possibility of taking $X^{*, \alpha}$ not necessarily positive, open the way for a natural generalization to the example of Section 4.2 even in the context of utility functions $u^{\alpha}$ that are not of a power type.

To get started, let us detail how it is possible to generate stochastic utilities from any family of utility functions $u^{\alpha}$ indexed by a parameter $\alpha$. The idea is the same as that of Proposition 4.1. Let $u^{\alpha}$ an utility function not necessary of power type and let $N_{t}^{(\alpha)}$ and $Z_{t}^{(\alpha)}$ two positive processes such that

$$
\frac{d N_{t}^{(\alpha)}}{N_{t}^{(\alpha)}}=\mu_{t}^{N,(\alpha)} d t+\delta_{t}^{N,(\alpha)} . d W_{t}, \quad \frac{d Z_{t}^{(\alpha)}}{Z_{t}^{(\alpha)}}=\mu_{t}^{Z,(\alpha)} d t+\delta_{t}^{Z,(\alpha)} \cdot d W_{t}, \quad Z_{0}=1 .
$$

Note that $Z^{\alpha}$ and $N^{\alpha}$ depend on the choice of $\alpha$. Next result, proved at first in Mrad (2009), generalizes Proposition 4.1. In particular, it gives sufficient conditions on the triplet $\left(u^{\alpha}, N^{(\alpha)}, Z^{(\alpha)}\right)$ under which the process $U^{\alpha}(t, x):=Z_{t}^{(\alpha)} u^{\alpha}\left(x / N_{t}^{(\alpha)}\right)$ is a consistent utility.

Proposition 5.1. Let $u^{\alpha}$ be a utility function.

(i) Assume that $N^{(\alpha)}$ is an admissible positive wealth process, i.e. $\left(\delta^{N,(\alpha)} \in \mathcal{R}, \mu^{N,(\alpha)}=r+\right.$ $\left.\eta . \delta^{N,(\alpha)}\right)$ and $Z^{(\alpha)}$ is a martingale such that $Z^{(\alpha)} X^{\kappa} / N^{(\alpha)}, \kappa \in \mathcal{R}$ is a local martingale for any $\kappa \in \mathbb{R}$. Then the process $U^{\alpha}$ defined by $U^{\alpha}(t, x)=Z_{t}^{(\alpha)} u^{\alpha}\left(x / N_{t}^{(\alpha)}\right)$ is a consistent stochastic utility with optimal wealth process $X^{\alpha, *}=N^{(\alpha)}$.

(ii) If $u^{\alpha}$ is a power or an exponential utility, then the condition " $Z^{(\alpha)}$ is martingale, $Z^{(\alpha)} X^{\kappa} / N^{(\alpha)}$ is a local martingale for any $\kappa \in \mathcal{R}$ " can be relaxed: 
a) If $u^{\alpha}$ is a power utility, the relaxed condition is given by Proposition 4.1.

b) If $u^{\alpha}$ is an exponential utility that is $u^{\alpha}(x)=\frac{1}{\alpha} e^{-\alpha x}$, it suffices to take $Z^{(\alpha)}$ and $N^{(\alpha)}$ such that

$$
\mu^{N,(\alpha)}=r+\sigma^{N,(\alpha)} . \eta, \quad \mu^{Z,(\alpha)}=\frac{1}{2}\left\|\eta-\delta^{N,(\alpha)}+\delta^{Z,(\alpha), \sigma}\right\|^{2}, \quad \delta^{N,(\alpha)} \in \mathcal{R} .
$$

In this case, the optimal policy $\kappa^{\alpha, *}$ is given by

$$
x \kappa_{t}^{\alpha, *}(x)=x \delta_{t}^{N,(\alpha)}+\frac{N_{t}^{(\alpha)}}{\alpha}\left(\eta_{t}-\delta_{t}^{N,(\alpha)}+\delta^{N,(\alpha), \sigma}\right) .
$$

Moreover, $x \mapsto x \kappa_{t}^{\alpha, *}(x)$ is a globally Lipchitz function.

In all cases $X^{*, \alpha}(x)$ is strictly increasing in $x$.

This result gives sufficient conditions under which $U^{\alpha}$, defined above, is a $\mathscr{X}$-consistent stochastic utility. Note also that this result generalizes the one in Musiela \& Zariphopoulou (2008) in the case of $u$ being an exponential utility.

In the following paragraphs, explicit illustrations are given, based on utilities functions of power and exponential type. Main tools are the results of Proposition 5.1.

\subsubsection{Example 1: Consistent utilities from optimal processes associated with power utilities functions}

In this paragraph, we are interested by applying the previous construction to the case where utilities $u^{\alpha}$ are of power type. For this, we consider a family $\left\{Z^{(\alpha)} ; \alpha>0\right\}$ such that $Z^{(\alpha)}$ satisfies, for each $\alpha$, the following dynamics

$$
\frac{d Z_{t}^{(\alpha)}}{Z_{t}^{(\alpha)}}=-\left((1-\alpha) r_{t}+\frac{1-\alpha}{2 \alpha}\left\|\eta_{t}+\delta_{t}^{Z,(\alpha), \sigma}\right\|^{2}\right) d t+\delta_{t}^{(\alpha)} \cdot d W_{t}, \quad Z(\alpha)_{0}=1 .
$$

Remark that contrary to the frame of Proposition 4.1, see Remark 4.1, the volatility vector $\delta^{Z,(\alpha)}$ depends on the parameter $\alpha$, which generalizes the construction of Section 4.2.

To ensure that $X^{*} Y^{*}$ is a martingale we make Assumption 4.1 in addition to (4.1), that is $\alpha \mapsto \frac{\delta^{(\alpha)}}{\alpha}$ is uniformly bounded.

According to Proposition 4.1 the process $U^{\alpha}(t, x)=Z_{t}^{(\alpha)} \frac{x^{1-\alpha}}{1-\alpha}$, is a $\mathscr{X}^{+}$-consistent dynamic utility such that the optimal policy $\kappa^{*}$ is given by $\kappa_{t}^{*}(x)=\frac{1}{\alpha}\left(\eta_{t}+\delta_{t}^{Z,(\alpha), \sigma}\right)$. Moreover, the optimal wealth process $X^{\alpha, *}$ and the optimal dual process $Y^{\alpha, *}$ associated with this power utility $U^{\alpha}$ are given by,

$$
\left\{\begin{array}{l}
X_{t}^{*, \alpha}(x)=x \bar{X}_{t}^{*, \alpha}=x e^{\int_{0}^{t}\left(r_{s}+\frac{1}{\alpha}\left(\eta_{s}+\delta_{s}^{Z,(\alpha), \sigma}\right) \cdot \eta_{s}\right) d s} \mathcal{E}_{t}\left(\frac{\eta+\delta^{Z,(\alpha), \sigma}}{\alpha}\right) \\
Y_{t}^{\alpha, *}(y)=y \bar{Y}_{t}^{\alpha, *}=y e^{-\int_{0}^{t} r_{s} d s} \mathcal{E}_{t}\left(\delta^{Z,(\alpha), \perp}-\eta\right)
\end{array}\right.
$$


From this, we consider the following processes $X^{*}$ and $Y^{*}$ given by

$$
\left\{\begin{array}{l}
X_{t}^{*}(x)=\int_{\mathbb{R}_{+}^{*}} x_{\alpha}(x) \bar{X}_{t}^{*, \alpha} m(d \alpha), \quad X_{0}^{*}(x)=x=\int_{\mathbb{R}_{+}^{*}} x_{\alpha}(x) m(d \alpha) \\
Y_{t}^{*}(y)=\int_{\mathbb{R}_{+}^{*}} y_{\alpha}(y) \bar{Y}_{t}^{\alpha, *} m(d \alpha), \quad Y_{0}^{*}(y)=y=\int_{\mathbb{R}_{+}^{*}} y_{\alpha}(x) m(d \alpha) .
\end{array}\right.
$$

By assumptions, $X^{*}$ is a wealth process and $Y^{*}$ is a state density process which are strictly increasing from 0 to $\infty$, we denote respectively $\mathcal{X}$ and $\mathcal{Y}$ their inverse with respect to their initial conditions. Consequently, for any utility function $u$ such that $x \mapsto Y^{*}\left(t, u_{x}(\mathcal{X}(t, z))\right)$ is integrable near to zero the process $U$ defined by

$$
U(t, x)=\int_{0}^{x} Y^{*}\left(t, u_{x}(\mathcal{X}(t, z))\right) d z
$$

is a consistent utility.

Although this class of utilities processes is simply generated from optimal processes associated with power utilities (the simplest utilities we can consider) nevertheless it is a richer class then that defined in Section 4.2 by equation (4.19) because here we have additional degree of freedom that is the family $\left\{y_{\alpha}, \alpha\right\}$.

\subsubsection{The case $x_{\alpha}(x)=x g(\alpha):$}

In this example, we are concerned with the particular case where the functions $x_{\alpha}(x)$ are linear with respect to the initial global wealth $x$, i.e., $x_{\alpha}(x)=x g(\alpha)$. This implies that the wealth process $X^{*}$ is linear with respect to its initial value $x$ and is given by

$$
X_{t}^{*}(x)=x \bar{X}_{t}^{*}, \bar{X}_{t}^{*}:=X_{t}^{*}(1)=\int_{\mathbb{R}_{+}^{*}} g(\alpha) \bar{X}_{t}^{\alpha, *} m(d \alpha) .
$$

In particular, we have the explicit formula for the inverse flow $\mathcal{X}$ of $X^{*}, \mathcal{X}_{t}(x)=x / X_{t}^{*}$.

Composing the stochastic flows $Y^{*}$ and $\mathcal{X}$, the derivative $U_{x}$ of the stochastic utility constructed above satisfies

$$
U_{x}(t, x)=Y_{t}^{*}\left(u_{x}\left(\mathcal{X}_{t}(x)\right)\right)=\int_{\mathbb{R}_{+}^{*}} y_{\alpha}\left(\bar{u}_{x}\left(\frac{x}{\bar{X}_{t}^{*}}\right)\right) \bar{Y}_{t}^{\alpha, *} m(d \alpha) .
$$

Integrating yields

$$
U(t, x)=\int_{\mathbb{R}_{+}^{*}}\left(\int_{0}^{x} y_{\alpha}\left(u_{x}\left(\frac{z}{\bar{X}_{t}^{*}}\right)\right) d z\right) \bar{Y}_{t}^{\alpha, *} m(d \alpha) .
$$

Moreover, if we take $y_{\alpha}(y)=y f(\alpha)$, then the utility processes $U$ rewrites, after integration with respect to $z$,

$$
U(t, x)=\bar{X}_{t}^{*} \int_{\mathbb{R}_{+}^{*}} f(\alpha) \bar{u}\left(\frac{x}{\bar{X}_{t}^{*}}\right) \bar{Y}_{t}^{\alpha, *} m(d \alpha)=\bar{X}_{t}^{*} \bar{Y}_{t}^{*} u\left(\frac{x}{X_{t}^{*}}\right)
$$


thus the utility process $U$ is simply the transformation of the utility function $u$ to a consistent one using the techniques of change of numeraire and probability: the numeraire $N$ is the optimal portfolio $\bar{X}^{*}$ and the change of probability $Z$ is the martingale $\bar{Y}^{*} \bar{X}^{*}$, which is in a perfect concordance with results of Proposition 5.1.

Particular form of the initial utility function $u$ : Let $\left\{u^{\alpha}, \alpha>0\right\}$ be a family of utilities functions (not necessarily of power type) and define the utility function $u$

$$
u_{x}(x):=\int_{\mathbb{R}_{+}^{*}} u_{x}^{\alpha}(x) m(d \alpha) .
$$

By definition $u_{x}$ is strictly decreasing with inverse $\left(u_{x}\right)^{-1}$, take $y_{\alpha}(y):=u_{x}^{\alpha}\left(\left(u_{x}\right)^{-1}(y)\right)$ and observe that

$$
y=\int_{\mathbb{R}_{+}^{*}} u_{x}^{\alpha}\left(\left(u_{x}\right)^{-1}(y)\right) m(d \alpha)=\int_{\mathbb{R}_{+}^{*}} y_{\alpha}(y) m(d \alpha) .
$$

Requirements of Theorem 2.1 being respected, the utility $U$ is given by

$$
U(t, x)=\int_{\mathbb{R}_{+}^{*}} \int_{0}^{x} u_{x}^{\alpha}\left(\frac{z}{\bar{X}_{t}^{*}}\right) d z \bar{Y}_{t}^{\alpha, *} m(d \alpha) .
$$

Integrating, yields

$$
U(t, x)=\bar{X}_{t}^{*} \int_{\mathbb{R}_{+}^{*}} u^{\alpha}\left(\frac{x}{\bar{X}_{t}^{*}}\right) \bar{Y}_{t}^{\alpha, *} m(d \alpha)
$$

which can be interpreted as the sum of consistent utilities $\bar{X}_{t}^{*} \bar{Y}_{t}^{\alpha, *} u^{\alpha}\left(\frac{x}{\bar{X}_{t}^{*}}\right)$ which are the transformation of the utilities $u^{\alpha}$ by the same change of numeraire $\bar{X}^{*}$ but with different change of probability defined by the martingale processes $\bar{X}_{t}^{*} \bar{Y}_{t}^{\alpha, *}$.

\subsubsection{The case $y_{\alpha}(y)=f(\alpha) y$}

In this example, we are concerned with the case where the initial conditions $y_{\alpha}(y)$ of the state price density processes $Y^{\alpha}$ are linear with respect to $y$. We then focus on the dual convex $\widetilde{U}$ of the utility $U$. The state price density process $Y^{*}$ is then linear with respect to $y$ and its inverse is given by $\left(Y_{t}^{*}\right)^{-1}(y)=\frac{y}{Y_{t}^{*}}$ where we recall $\bar{Y}_{t}^{*}:=Y_{t}^{*}(1)=\int_{\mathbb{R}_{+}^{*}} f(\alpha) Y_{t}^{\alpha, *} m(d \alpha)$. The dual convex conjugate $\widetilde{U}$ of $U$ becomes

$$
\begin{aligned}
\widetilde{U}(t, y) & =\int_{y}^{+\infty} X_{t}^{*}\left(\left(Y_{t}^{*}\right)^{-1}\left(\left(u_{x}\right)^{-1}(z)\right)\right) d z \\
& =\int_{\mathbb{R}_{+}^{*}}\left[\int_{y}^{+\infty} x_{\alpha}\left(\left(u_{x}\right)^{-1}\left(\frac{z}{\bar{Y}_{t}^{*}}\right)\right) d z\right] X_{t}^{*, \alpha} m(d \alpha) .
\end{aligned}
$$


Particular form of the initial utility function $u$ : Let $\left\{u^{\alpha}, \alpha>0\right\}$ be a family of utilities functions (not necessarily of power type) and define the utility function $u$ via the derivative of its conjugate by

$$
-\tilde{u}_{y}(y):=\left(u_{x}\right)^{-1}(y)=\int_{\mathbb{R}_{+}^{*}}\left(u_{x}^{\alpha}\right)^{-1}(y) m(d \alpha) .
$$

By definition $\left(u_{x}\right)^{-1}$ is strictly increasing with inverse $u_{x}$, take $x_{\alpha}(x):=\left(u_{x}^{\alpha}\right)^{-1}\left(u_{x}(x)\right)$ and observe that

$$
x=\int_{\mathbb{R}_{+}^{*}}\left(u_{x}^{\alpha}\right)^{-1}\left(u_{x}(y)\right) m(d \alpha)=\int_{\mathbb{R}_{+}^{*}} x_{\alpha}(x) m(d \alpha) .
$$

Requirements of Theorem 2.1 being respected, the convex conjugate $\widetilde{U}$ of $U$ is given by

$$
\widetilde{U}(t, y)=\int_{\mathbb{R}_{+}^{*}}\left[\int_{y}^{+\infty}\left(u_{x}^{\alpha}\right)^{-1}\left(\frac{z}{\bar{Y}_{t}^{*}}\right) d z\right] \bar{X}_{t}^{*, \alpha} m(d \alpha) .
$$

Denoting by $\tilde{u}$ the convex conjugate of $u$, integrating yields

$$
\widetilde{U}(t, y)=\bar{Y}_{t}^{*} \int_{\mathbb{R}_{+}^{*}} \tilde{u}^{\alpha}\left(\frac{z}{\bar{Y}_{t}^{*}}\right) \bar{X}_{t}^{*, \alpha} m(d \alpha)
$$

which is interpreted as the sum of the convex conjugate $\bar{Y}_{t}^{*} \bar{X}_{t}^{*, \alpha} \tilde{u}^{\alpha}\left(\frac{z}{\bar{Y}_{t}^{*}}\right)$ of consistent utilities $\bar{X}_{t}^{*} \bar{Y}_{t}^{\alpha, *} u^{\alpha}\left(\frac{x}{\bar{X}_{t}^{*}}\right)$ in (5.26), which are the transformation of the utilities $u^{\alpha}$ by the same change of numeraire $\bar{X}^{*}$ but with different change of probability defined by the martingale processes $\bar{X}_{t}^{*} \bar{Y}_{t}^{\alpha, *}$.

The decreasing consistent utilities are easily obtained by considering

$$
\left(u_{x}\right)^{-1}(x)=\int_{\mathbb{R}_{+}^{*}} x^{-\frac{1}{\alpha}} m(d \alpha), x_{\alpha}=\left[\left(u_{x}\right)^{-1}\right]^{-\frac{1}{\alpha}}, y_{\alpha}(y)=y f(\alpha)
$$

and taking $r \equiv 0, \delta^{Z,(\alpha)} \equiv 0$.

\subsubsection{Example 2: Consistent utilities from optimal processes associated with exponential utilities functions}

The proof of Theorem 2.1 is based on the key identity $U_{x}\left(t, X_{t}^{*}(x)\right)=Y_{t}^{*}\left(u_{x}(x)\right)$, on the monotonicity of the optimal processes and on the orthogonality conditions between these processes. This theorem can be easily extended to the context of utilities defined on whole $\mathbb{R}$ which are consistent with a class of processes $\mathscr{X}$ bounded by below (ensuring integrability). As a result, we can place ourselves in the context of exponential utilities and generate by the same formula (2.7) a new class of dynamic utilities. This is the purpose of this section.

In this section $u^{\alpha}$ is an exponential utility with risk aversion $\alpha$ that is $u^{\alpha}(x)=1-\frac{1}{\alpha} e^{-\alpha x}$. As negative wealths are allowed, the dynamics (4.2) is replaced by

$$
d X_{t}^{\kappa}=X_{t}^{\kappa} r_{t} d t+\kappa_{t} \cdot\left(d W_{t}+\eta_{t} d t\right)
$$


According to Proposition 5.1, the numeraire $N^{\alpha}$ and the process $Z^{\alpha}$ are solutions of the following dynamics

$$
\left\{\begin{array}{l}
\frac{d N_{t}^{(\alpha)}}{N_{t}^{(\alpha)}}=\left(r_{t}+\delta_{t}^{N,(\alpha)} \cdot \eta_{t}\right) d t+\delta_{t}^{N,(\alpha)} \cdot d W_{t}, \quad \delta_{t}^{N,(\alpha)} \in \mathcal{R}_{t}, \quad t \geq 0, \quad N_{0}=1 \\
\frac{d Z_{t}^{(\alpha)}}{Z_{t}^{(\alpha)}}=\frac{1}{2}\left\|\eta_{t}-\delta_{t}^{N,(\alpha)}+\delta_{t}^{Z,(\alpha), \sigma}\right\|^{2} d t+\delta_{t}^{Z,(\alpha)} \cdot d W_{t}, \quad t \geq 0, \quad Z_{0}=1
\end{array}\right.
$$

To ensure that $X^{*} Y^{*}$ is a martingale we make the following assumption

Assumption 5.1. The minimal risk prime $\eta$ and the volatility vectors $\delta^{N,(\alpha)}, \delta^{Z,(\alpha)}, \alpha \in \mathbb{R}_{+}^{*}$ are uniformly bounded.

According to Proposition 5.1, the optimal policy $x \kappa^{*}$ is given by

$$
x \kappa_{t}^{\alpha, *}(x)=x \delta_{t}^{N,(\alpha)}+\frac{N_{t}^{(\alpha)}}{\alpha}\left(\eta_{t}-\delta_{t}^{N,(\alpha)}+\delta^{Z,(\alpha), \sigma}\right) .
$$

In turn, the optimal portfolio $X^{*, \alpha}$ satisfies

$$
d X_{t}^{*, \alpha}(x)=r_{t} X_{t}^{*, \alpha}(x) d t+\left(X_{t}^{*, \alpha}(x) \delta_{t}^{N,(\alpha)}+\frac{N_{t}^{(\alpha)}}{\alpha}\left(\eta_{t}-\delta_{t}^{N,(\alpha)}+\delta^{Z,(\alpha), \sigma}\right)\right) \cdot\left(d W_{t}+\eta_{t} d t\right)
$$

Applying Itô's formula to the process $\frac{X_{t}^{*}(x)}{N_{t}^{(\alpha)}}$, simple calculations lead to

$$
d \frac{X_{t}^{*, \alpha}(x)}{N_{t}^{(\alpha)}}=\frac{1}{\alpha}\left(\eta_{t}-\delta_{t}^{N,(\alpha)}+\delta_{t}^{Z,(\alpha), \sigma}\right) \cdot\left(d W_{t}+\left(\eta_{t}-\delta_{t}^{N,(\alpha)}\right) d t\right),
$$

which is equivalent to

$$
X_{t}^{\alpha, *}\left(x_{\alpha}(x)\right)=N_{t}^{(\alpha)}\left[x_{\alpha}(x)+\frac{1}{\alpha} \int_{0}^{t}\left(\eta_{s}-\delta_{s}^{N,(\alpha)}+\delta_{s}^{Z,(\alpha), \sigma}\right) \cdot\left(d W_{s}+\left(\eta_{s}-\delta_{s}^{N,(\alpha)}\right) d s\right)\right] .
$$

Consequently the global wealth process writes

$$
\begin{aligned}
& X_{t}^{*}(x)=\int_{\mathbb{R}_{+}^{*}} X_{t}^{*, \alpha}\left(x_{\alpha}(x)\right) m(d \alpha) \\
= & {\left[\int_{\mathbb{R}_{+}^{*}} x_{\alpha}(x) N_{t}^{(\alpha)} m(d \alpha)\right.} \\
+ & \int_{\mathbb{R}_{+}^{*}} N_{t}^{(\alpha)}\left(\frac{1}{\alpha} \int_{0}^{t}\left(\eta_{s}-\delta_{s}^{N,(\alpha)}+\delta_{s}^{Z,(\alpha), \sigma}\right) \cdot\left(d W_{s}+\left(\eta_{s}-\delta_{s}^{N,(\alpha)}\right) d s\right) m(d \alpha)\right] .
\end{aligned}
$$

To achieve the construction in this exponential framework we will also give an explicit form to $Y^{*}$. For this, we begin by calculating $Y^{\alpha, *}$ before integrating with respect to $\alpha$ and the measure $m$. From previous equations, it follows

$$
e^{-\alpha \frac{X_{t}^{*, \alpha}(x)}{N_{t}^{(\alpha)}}}=e^{-\alpha x-\int_{0}^{t}\left(\eta_{s}-\delta_{s}^{N,(\alpha)}+\delta_{s}^{Z,(\alpha), \sigma}\right) \cdot\left(d W_{s}+\left(\eta_{s}-\delta_{s}^{N,(\alpha)}\right) d s\right)} .
$$


Multiplying by $Z_{t}^{(\alpha)}$, one can easily obtain

$$
Z_{t}^{(\alpha)} e^{-\alpha \frac{X_{t}^{*, \alpha}(x)}{N_{t}^{(\alpha)}}}=e^{-\alpha x} \mathcal{E}_{t}\left(\delta^{Z,(\alpha), \perp}-\eta\right)
$$

which implies

$$
Y_{t}^{\alpha, *}(y):=U_{x}^{\alpha}\left(t, X_{t}^{*, \alpha}\left(\left(u_{x}^{\alpha}\right)^{-1}(y)\right)\right)=\frac{Z_{t}^{(\alpha)}}{N_{t}^{(\alpha)}} e^{-\alpha \frac{X_{t}^{*, \alpha}\left(\left(u_{x}^{\alpha}\right)^{-1}(y)\right)}{N_{t}^{(\alpha)}}}=y Y_{t}^{\delta^{Z,(\alpha), \perp}}
$$

where $Y^{\delta^{Z,(\alpha), \perp}}$ denote the state price density process given by (4.4) for $\nu=\delta^{Z,(\alpha), \perp}$ and with initial value equal to 1 . Integrating with respect to $\alpha$, the process $Y^{*}(y)$ is given by

$$
Y_{t}^{*}(x)=\int_{\mathbb{R}_{+}^{*}} y_{\alpha}(y) Y_{t}^{\delta^{Z,(\alpha), \perp}} m(d \alpha) .
$$

Case $N^{\alpha}=N$ : We are now concerned with the particular case where the process $N^{\alpha}$ does not depend on $\alpha$ and is equal to $N$. Using $\delta^{N,(\alpha)}=\delta^{N}$, it is immediate that the global wealth process $X^{*}$ is given by

$$
X_{t}^{*}(x)=N_{t}\left[x+\int_{\mathbb{R}_{+}^{*}} \frac{1}{\alpha} \int_{0}^{t}\left(\eta_{s}-\delta_{s}^{N}+\delta_{s}^{Z,(\alpha), \sigma}\right) \cdot\left(d W_{s}+\left(\eta_{s}-\delta_{s}^{N}\right) d s\right) m(d \alpha)\right],
$$

where we use the identity $X_{0}^{*}(x)=x=\int_{\mathbb{R}_{+}^{*}} x_{\alpha}(x) m(d \alpha)$ (see equation (4.14)). Hence, $X^{*}$ is strictly increasing with respect to $x$ with inverse $\mathcal{X}$ given by,

$$
\mathcal{X}_{t}(x)=\frac{x}{N_{t}}-M_{t}^{\alpha}
$$

where

$$
M_{t}^{(\alpha)}=\int_{\mathbb{R}_{+}^{*}} \frac{1}{\alpha} \int_{0}^{t}\left(\eta_{s}-\delta_{s}^{N}+\delta_{s}^{Z,(\alpha), \sigma}\right) \cdot\left(d W_{s}+\left(\eta_{s}-\delta_{s}^{N}\right) d s\right) m(d \alpha) .
$$

Let $u: \mathbb{R} \mapsto \mathbb{R}$ be a utility function with good integrability conditions. Composing the stochastic flows $Y^{*}, u_{x}$ and $\mathcal{X}$, the derivative $U_{x}$ of the stochastic utility constructed satisfies

$$
U_{x}(t, x)=Y_{t}\left(u_{x}\left(\mathcal{X}_{t}(x)\right)\right)=\int_{\mathbb{R}_{+}^{*}} y_{\alpha}\left(u_{x}\left(\frac{x}{N_{t}}-M_{t}^{(\alpha)}\right)\right) Y_{t}^{\delta^{Z,(\alpha), \perp}} m(d \alpha) .
$$

Integrating yields

$$
U(t, x)=\int_{\mathbb{R}_{+}^{*}}\left[\int_{0}^{x} y_{\alpha}\left(u_{x}\left(\frac{z}{N_{t}}-M_{t}^{(\alpha)}\right)\right) d z\right] Y_{t}^{\delta^{Z,(\alpha), \perp}} m(d \alpha)
$$

Observe, in this particular case, that the functions $x_{\alpha}$ do not play any role in the construction of $U$. 
$\checkmark$ Case where $y_{\alpha}(y)=y$ : the last identity becomes

$$
U(t, x)=N_{t} \int_{\mathbb{R}_{+}^{*}} u\left(\frac{z}{N_{t}}-M_{t}^{(\alpha)}\right) Y_{t}^{\delta^{Z,(\alpha), \perp}} m(d \alpha) .
$$

$\checkmark$ Case where $y_{\alpha}(y)=e^{-\alpha\left(u_{x}\right)^{-1}(y)}$, we get

$$
U(t, x)=N_{t} \int_{\mathbb{R}_{+}^{*}}\left(1-\frac{1}{\alpha} e^{-\alpha \frac{x}{N_{t}}+\alpha M_{t}^{(\alpha)}}\right) Y_{t}^{\delta^{Z,(\alpha), \perp}} m(d \alpha) .
$$

\subsubsection{Example 3: Consistent utilities from optimal processes associated with dif- ferent types of utilities functions}

In the examples above consistent utilities are generated from a family of utility functions $u^{\alpha}$ either power type or exponential type. The naturally question arising is if one can mix different types of utilities. The answer to this question is in fact positive. Indeed the parameter $\alpha$ plays the role of a simple index that does not necessarily refer to risk aversion coefficient. Besides, an intuitive explanation for this construction to be valid is the fact that despite the initial functions $u^{\alpha}$ are of a different types the optimal processes $\left(X^{\alpha, *}\right)_{\alpha \in \mathbb{R}_{+}^{*}}$ and $\left(Y^{\alpha, *}\right)_{\alpha \in \mathbb{R}_{+}^{*}}$ are such that $\left(X^{\alpha, *} Y^{\alpha^{\prime}, *}\right)_{\alpha \neq \alpha^{\prime}}$ are martingales (portfolios versus state price density processes). Assuming uniform integrability assumptions of the diffusion parameters of $Z^{\alpha}$ and $N^{\alpha}$, the strictly increasing processes $X^{*}$ and $Y^{*}$ are such that $X^{*} Y^{*}$ is a martingale, which is sufficient to apply the general construction result given by Theorem 2.1.

Example Herein, we give an example based on a mixture of power and exponential utilities. Consider the measure $m$ given by $\lambda \delta_{\alpha_{1}}+(1-\lambda) \delta_{\alpha_{1}}$, where $\delta_{\alpha}$ is the Dirac measure. Let also $u^{\alpha_{1}}$ of a power type and $u^{\alpha_{2}}$ of an exponential type and consider the process

$$
M_{t}^{\left(\alpha_{2}\right)}:=\int_{0}^{t}\left(\eta_{s}-\delta_{s}^{N,\left(\alpha_{2}\right)}+\delta_{s}^{Z,\left(\alpha_{2}\right), \sigma}\right) \cdot\left(d W_{s}+\left(\eta_{s}-\delta_{s}^{N,\left(\alpha_{2}\right)}\right) d s\right) .
$$

By Proposition 4.1 and Proposition 5.1, we know that

$$
\left\{\begin{array}{l}
X_{t}^{*, \alpha_{1}}(x)=x X_{t}^{*, \alpha_{1}}, \quad X_{t}^{\alpha_{2}, *}(x)=N_{t}^{\left(\alpha_{2}\right)}\left[x+M_{t}^{\left(\alpha_{2}\right)}\right] \\
Y_{t}^{\alpha_{1}, *}(y)=y Y_{t}^{\delta^{Z,\left(\alpha_{1}\right), \perp}}, \quad Y_{t}^{\alpha_{2}, *}(y)=y Y_{t}^{\delta^{Z,\left(\alpha_{2}\right), \perp}}
\end{array}\right.
$$

with $X_{t}^{*, \alpha_{1}}$ given by (5.16), consequently

$$
\left\{\begin{array}{l}
X_{t}^{*}(x)=x\left(\lambda X_{t}^{*, \alpha_{1}}+(1-\lambda) N_{t}^{\left(\alpha_{2}\right)}\right)+(1-\lambda) M_{t}^{\left(\alpha_{2}\right)} N_{t}^{\left(\alpha_{2}\right)} \\
Y_{t}^{*}(y)=y\left(\lambda Y_{t}^{\delta^{Z,\left(\alpha_{1}\right), \perp}}+(1-\lambda) Y_{t}^{\delta^{Z,\left(\alpha_{2}\right), \perp}}\right) .
\end{array}\right.
$$

Denoting by $X^{\lambda}$ and $Y^{\lambda}$ the processes

$$
\left\{\begin{array}{c}
X^{\lambda}:=\left(\lambda X_{t}^{*, \alpha_{1}}+(1-\lambda) N_{t}^{\left(\alpha_{2}\right)}\right) \\
Y^{\lambda}:=\left(\lambda Y^{\delta^{Z,\left(\alpha_{1}\right), \perp}}+(1-\lambda) Y^{\delta^{Z,\left(\alpha_{2}\right), \perp}}\right)
\end{array}\right.
$$


and assuming them to be a.s. non null, ensure that $X^{*}(x)$ and $Y^{*}(y)$ are strictly monotonous with respect to their initial conditions, with inverse flows

$$
\mathcal{X}_{t}(x)=\frac{x-(1-\lambda) M_{t}^{\left(\alpha_{2}\right)} N_{t}^{\left(\alpha_{2}\right)}}{X_{t}^{\lambda}}, \quad \mathcal{Y}_{t}(y)=\frac{y}{Y_{t}^{\lambda}} .
$$

Let $u$ a utility function defined on $\mathbb{R}$, by Theorem 2.1 , the progressive utility $U$ defined by

$$
U(t, x)=Y_{t}^{\lambda} \int_{0}^{x} u_{x}\left(\frac{z-(1-\lambda) M_{t}^{\left(\alpha_{2}\right)} N_{t}^{\left(\alpha_{2}\right)}}{X_{t}^{\lambda}}\right) d z,
$$

is a $\mathscr{X}$-consistent utility.

Conclusion Several contributions to the study of dynamic (forward) utilities are given in this paper. In Section 3, we characterize, as a sup-convolution, the consistent utilities whose dual processes are an aggregation of dual convex utilities under the Pareto optimality condition which imposes a common optimal dual process $Y^{*}$.

But, going beyond the Pareto-optimality, the main contribution of this paper is given in Sections 4.2 and 5. The key point of the construction is to argue directly in terms of the optimal wealth and dual process and not in terms of consistent utilities for the simple reason that the sum of two admissible wealths (state price) is always an admissible wealth while the sum of two consistent utilities is not a consistent utility, except in the very particular case where optimal wealths are identical and optimal dual processes are also identical. Note also that the fact that $X^{\alpha, *} Y^{\alpha^{\prime}, *}, \alpha \neq \alpha^{\prime}$ are martingales, plays a crucial role in the proposed construction. Without this assumption, the global wealth process $X^{*}$ and the global state density price $Y^{*}$ do not satisfy the necessary martingale condition.

The time-decreasing utilities are only a special case of the family of dynamic utilities built in Sections 3, 4.2 and 5. The new proof of Theorem 4.2, which we propose is also a nice contribution, simple, educational and requires no additional results to show that these utilities are mixtures of power ones.

\section{References}

P. Barrieu \& N. El Karoui (2005) Inf-convolution of risk measures and optimal risk transfer. In : Finance and Stochastics 9 (2), 269-298. George Stoica.

F. P. Y. S. Berrier, L. C. G. Rogers \& M. Tehranchi (2009). A characterization of forward utility functions. Preprint, 21.

T. Choulli, C. Stricker \& J. Li (2007) Minimal Hellinger martingale measures of order $q$. In : Finance and Stochastics 11 (3),399-427. 
Censor, Y. (1977). Pareto optimality in multiobjective problems. In Applied Mathematics and Optimization, 4(1), 41-59.

F. Delbaen \& W. Schachermayer (1994) A general version of the fundamental theorem of asset pricing. In : Mathematische Annalen 300 (3), 463-520.

F. Delbaen \& W. Schachermayer (1998) The fundamental theorem of asset pricing for unbounded stochastic processes. In : Mathematische Annalen 312 (2), 215-250.

N. El Karoui \& M. Mrad (2013) An exact connection between two solvable SDEs and a non linear utility stochastic PDEs. In : SIAM Journal on Financial Mathematics 4 (1), 697-736.

N. El Karoui, C. Hillairet, \& M. Mrad (2018) Consistent utility of investment and consumption : a forward/backward SPDE viewpoint. In : Stochastics 90 (6), 927-954. Taylor \& Francis.

N. El Karoui, C. Hillairet, \& M. Mrad(2014) Affine long term yield curves : an application of the Ramsey rule with progressive utility. In : Journal of Financial Engineering 1 (1).

N. El Karoui \& M. Mrad (2019) Indifference and Marginal Indifference Pricing Using Consistent Stochastic Utilities. Preprint.

N. El Karoui, C. Hillairet, \& M. Mrad (2019) Ramsey rule with progressive utility for long term yield curves Modeling. arXiv:1404.1895, preprint (submitted for publication).

N. El Karoui \& M. Mrad (2020) Recover dynamic utility from observable process: Application to the economic equilibrium. HAL hal-01966312v3, preprint (To appear in SIAM JOURNAL).

E. Gobet \& M. Mrad (2018) Convergence rate of strong approximations of compound random maps, application to SPDEs. In Discrete $\&$ Continuous Dynamical Systems-B 23 (10), 4455. American Institute of Mathematical Sciences.

H. He and H. Leland (1993) On equilibrium asset price processes. In : The Review of Financial Studies 6 (3), 593-617. Oxford University Press.

V. Henderson \& D. Hobson (2007) Horizon-unbiased utility functions. In : Stochastic Processes and their Applications 117 (11), 1621-1641.

I. Karatzas \& S.E. Shreve (2001) Methods of Mathematical Finance. In : Hardcover, Springer Annalen.

M. Majumdar, McFadden, D. \& Mitra, T. (1980). Pareto optimality and competitive equilibrium in infinite horizon economies. In : Journal of Mathematical Economics, 7(1), 1-26. 
A. Migdalas, P. M. Pardalos \& L. Pitsoulis (2008). Pareto optimality, game theory and equilibria (Vol. 17).In: Springer Science \&5 Business Media 17.

M. Mrad (2009) Utilités Progressives Dynamiques. PhD Thesis, Department of Applied Mathematics, Ecole Polytechnique X.

M. Musiela \& T. Zariphopoulou (2008). Optimal asset allocation under forward exponential ferformance Criteria. In : Contemp. Math., 4, 285-300, Institute of Mathematical Statistics.

M. Musiela \& T. Zariphopoulou (2011). Initial investment choice and optimal future allocations under time-monotone performance criteria. In : International Journal of Theoretical and Applied Finance, 14 (1), 61-81,World Scientific.

M. Musiela \& T. Zariphopoulou (2003). Backward and forward utilities and the associated pricing systems: The case study of the binomial model. Preprint.

M. Musiela \& T. Zariphopoulou (2007). Stochastic partial differential equations in portfolio choice. Preliminary report.

M. Musiela \& T. Zariphopoulou(2004). An example of indifference prices under exponential preferences. In : Finance and Stochastics 8 (2), 229-239.

M. Musiela \& T. Zariphopoulou (2010). Portfolio choice under space-time monotone performance criteria. In : SIAM Journal on Financial Mathematics 1 (1), 326-365. SIAM.

V. Pareto (1913). Il massimo di utilità per una collettività in sociologia. In: Giornale degli economisti e rivista di statistica, 46 (4), 337-341, JSTOR.

D. V. Widder (1963) The role of the Appell transformation in the theory of heat conduction. In : Transactions of the American Mathematical Society 109, 121-134. I. I. Hirschman, Jr.

D. V. Widder (1975) The Heat Equation. In : Pure and Applied Mathematics 67, Academic Press [Harcourt Brace Jovanovich Publishers]. Gerald G. Bilodeau.

G. Zitkovic (2008). A dual characterization of self-generation and log-affine forward performances. Preprint. 